\title{
1 DUX4 regulates oocyte to embryo transition in human
}

3 Sanna Vuoristo ${ }^{1, \#^{*}}$, Christel Hydén-Granskog ${ }^{3}$, Masahito Yoshihara ${ }^{1}$, Shruti

4 Bhagat $^{1,4}$, Lisa Gawriyski ${ }^{5}$, Eeva-Mari Jouhilahti ${ }^{6}$, Anastassius Damdimopoulos $^{7}$,

$5 \quad$ Vipin Ranga ${ }^{8}$, Mahlet Tamirat $^{8}$, Mikko Huhtala ${ }^{8}$, Kosuke Hashimoto ${ }^{4}$, Kaarel

6 Krjutškov ${ }^{1,6,9,10}$, Gaëlle Recher ${ }^{11}$, Sini Ezer ${ }^{6,12}$, Priit Paluoja ${ }^{9,13}$, Pauliina Paloviita ${ }^{2}$,

$7 \quad$ Yujiro Takegami ${ }^{14}$, Ai Kanemaru ${ }^{14}$, Karolina Lundin², Tomi Airenne ${ }^{8}$, Timo

8 Otonkoski $^{6,15}$, Juha S. Tapanainen ${ }^{2,3,16}$, Hideya Kawaji ${ }^{4,17,18}$, Yasuhiro Murakawa ${ }^{4,19}$,

9 Thomas R. Bürglin ${ }^{20}$, Markku Varjosalo ${ }^{5}$, Mark S. Johnson ${ }^{8}$, Timo Tuuri ${ }^{2,}$, , Shintaro

10 Katayama $^{1,12^{*}}$ and Juha Kere ${ }^{1,6,12^{*}}$

$12{ }^{1}$ Karolinska Institutet, Department of Biosciences and Nutrition, Huddinge, Sweden

$13{ }^{2}$ University of Helsinki, Department of Obstetrics and Gynecology, Helsinki, Finland

$14{ }^{3}$ Helsinki University Hospital, Department of Obstetrics and Gynecology, Helsinki,

15 Finland

${ }^{4}$ RIKEN Center for Integrative Medical Sciences, Yokohama, Japan

$17{ }^{5}$ University of Helsinki, Institute of Biotechnology, Helsinki, Finland

$19{ }^{7}$ Karolinska Institutet, Bioinformatics and Expression Analysis Core Facility,

20 Huddinge, Sweden

$21{ }^{8}$ Structural Bioinformatics Laboratory, Biochemistry, Faculty of Science and

22 Engineering, Åbo Akademi University, Turku, Finland 
$23{ }^{9}$ Competence Centre for Health Technologies, Tartu, Estonia

$24{ }^{10}$ Department of Obstetrics and Gynecology, Institute of Clinical Medicine, University

25 of Tartu; Tartu, Estonia

$26 \quad{ }^{11}$ Institut d'Optique Graduate School, CNRS - Université de Bordeaux, Talence, France

$27 \quad{ }^{12}$ Folkhälsan Research Center, Helsinki, Finland

$28 \quad{ }^{13}$ Institute of Computer Science, University of Tartu, Tartu, Estonia

$29 \quad{ }^{14}$ K.K. DNAFORM, Yokohama, Japan

$30 \quad{ }^{15}$ Children's Hospital, Helsinki University Hospital and University of Helsinki, Finland

$31 \quad{ }^{16}$ Oulu University Hospital, Oulu, Finland

$32 \quad{ }^{17}$ RIKEN Preventive Medicine and Diagnosis Innovation Program, Wako, Japan

$33{ }^{18}$ Tokyo Metropolitan Institute of Medical Science, Tokyo, Japan

$34{ }^{19}$ IFOM, The FIRC Institute of Molecular Oncology, Milan, Italy

$35{ }^{20}$ University of Basel, Department of Biomedicine, Basel, Switzerland

$38 \quad$ \#Current affiliation

$39 \quad{ }^{*}$ Corresponding author 


\section{Abstract}

46 During the human oocyte-to-embryo transition, the fertilized oocyte undergoes

47 final maturation and the embryo genome is gradually activated during the first

48 three cell divisions. How this transition is coordinated in humans is largely

49 unknown. We show that the double homeodomain transcription factor DUX4

50 contributes to this transition. DUX4 knockdown in human zygotes caused

51 insufficient transcriptome reprogramming as observed three days after

52 fertilization. Induced DUX4 expression in human embryonic stem cells activated

53 transcription of thousands of newly identified bi-directional transcripts, including

54 putative enhancers for embryonic genome activation genes such as LEUTX. DUX4

55 protein interacted with transcriptional modifiers that are known to couple

56 enhancers and promoters. Taken together, our results reveal that DUX4 is a

57 pioneer regulating oocyte-to-embryo transition in human through activation of

58 intergenic genome, especially enhancers, and hence setting the stage for early

59 human embryo development.

60

61

62 
66 Mammalian pre-implantation development commences with oocyte-to-embryo

67 transition, which involves fundamental changes in the epigenetic landscapes,

68 modulation of cell cycle control, and translation or clearance of selected maternal

69 mRNAs, culminating to embryonic genome activation ${ }^{1,2}$. The pioneer regulators

70 orchestrating the oocyte-to-embryo transition and first embryo genome activation steps

71 in human remain poorly understood. The conserved double homeodomain transcription

72 factor DUX4 represents a plausible candidate regulating the oocyte-to-embryo

73 transition in humans, given its capacity to activate germline genes and genomic repeat

74 elements ${ }^{3-5}$. Here we show that DUX4 is able to launch the first reprogramming steps

75 from oocyte to embryo in human by activating thousands of novel enhancers and

76 therein, modulating the transcriptome and chromatin. Human DUX4 knockdown

77 embryos are viable until the third day of development, but their transcriptome is

78 severely altered. Our proteomics approaches suggest that DUX4 binds to Mediator

79 complex and chromatin modifiers through its C-terminal domains, providing a likely

80 explanation to how DUX4 may extensively modulate the genome. This study implies a

81 wider role for $D U X 4$ as a cellular gate keeper acting both as a general genomic modifier

82 of cell fate as well as a specific inducer of first wave embryo genome activation genes.

\section{$84 \quad$ Results}

Quantification of DUX4 in human embryos

The DUX4 induced gene network is highly conserved ${ }^{6}$ and recent reports showed that

87 DUX4 is expressed in early human embryos ${ }^{3,4}$. However, details of this dynamic 88 process, including initiation of $D U X 4$ expression, remained ambiguous. Therefore, we 

in zygotes, while few transcripts were found in MII oocytes or cleavage embryos ${ }^{7}$ (Fig. 1b). Induction of the $D U X 4$ mRNA orthologues in mouse and non-human primate zygotes is evolutionary conserved (Extended Data Fig.1a). Antibody staining revealed that DUX4 protein is highly abundant in the cytoplasm and nuclei of zygotes as well as 2-cell and 4-cell embryos (Fig. 1c). Quantification of the nuclear DUX4 staining intensities in $3 \mathrm{D}$ showed a variable but increasing nuclear signal from the zygotes up to 4-cell embryos, while almost no signal was detected in 8-cell embryos (Fig 1c, d). In one single very early 8 -cell stage embryo there was high variability in the nuclear DUX4 staining, consistent with rapid clearance of the DUX4 protein (Extended Data

101 Fig. 1b). These results demonstrate that DUX4 transcripts appear around the time of

102 fertilisation and is followed by cytoplasmic and nuclear localisation of the DUX4 103 protein during the first two days of human embryo development coinciding with the

104 timeframe of the oocyte-to-embryo transition and activation of the genome.

107 Given the short-term and precise manifestation of DUX4 mRNA and protein in human

108 zygotes and early cleavage stage embryos, we next asked how DUX4 regulates the first

109 steps of human embryo development. We microinjected either DUX4 targeting siRNA

110 (siDUX4) or control siRNA (siCTRL) into triploid human zygotes and followed their

111 development for $48 \mathrm{~h}$ after the microinjections, until the third day of development (Fig.

112 1e). Staining of the DUX4 protein was very faint or absent in the siDUX4 embryos but 113 strongly positive in the siCTRL embryos $24 \mathrm{~h}$ after microinjection (Fig. 1f), confirming 
114 that the $D U X 4$ targeting siRNA efficiently down-regulated $D U X 4$ expression. The cells

115 from the microinjected embryos were collected $48 \mathrm{~h}$ after microinjections and

116 sequenced for identification of transcript far 5'-ends (TFEs), which represent

117 transcription start sites of polyA-tailed RNAs ${ }^{8}$. Group-wise comparison suggested that

118 a number of TFEs were upregulated in the siDUX4 embryos (Fig. 1g, Supplementary

119 Information 1). We annotated the upregulated TFEs and compared them to our

120 published gene expression data $\operatorname{set}^{7,8}$ on human MII oocytes, zygotes, and cleavage

121 cells. These analyses revealed that a large number of mRNAs enriched in siDUX4

122 embryos were normally down-regulated during transition from oocytes or zygotes to 4-

123 cell embryos (Fig. 1h, i) and from 4-cell embryos to 8-cell embryos (Fig 1j). The most

124 differentially expressed genes between the siCTRL and siDUX4 blastomeres were

125 maternal genes, such as $G D F 9^{9}$ and $Z P 1$ and $Z P 2^{10}$ (Fig. 1k). In agreement with the

126 presence of maternal transcripts, gene expression enrichment analysis using TopAnat ${ }^{11}$

127 for the genes retained in the siDUX4 embryos resulted in terms such as 'female germ

128 cell' and 'oocyte' (Extended Data Fig. 2a). Thus, maternal genes that normally undergo

129 targeted clearance during the oocyte-to-embryo transition were retained after the

130 knock-down of DUX4 in human zygotes. Investigating further, we identified 3,196

131 TFEs that are highly variable between the microinjected blastomeres. Weighted

132 correlation network analysis (WGCNA) ${ }^{12}$ classified these variable TFEs into three

133 modules: TFEs in blue and brown modules overlapped with the upregulated TFEs

134 during normal pre-implantation development ${ }^{8}$, suggesting embryonic genome

135 activation modules, while the TFEs in the turquoise module were associated with

136 maternal genes (Extended Data Fig. 2b). According to the representative expression

137 pattern, siDUX4 blastomeres did not upregulate the genome activation module TFEs

138 (Extended Data Fig. 2c), suggesting insufficient activation of their genome due to 
$139 D U X 4$ knock-down. Although retroelement-derived transcription by DUX4 binding has

140 been reported ${ }^{13}$, only a few genome activation module TFEs overlapped with the 56

141 families of repeat elements and known DUX4 binding sites (Extended Data Fig. 2d).

142 Surprisingly, TFEs in the maternal turquoise module overlapped with ERVL/ERVL-

143 MaLR elements and the known DUX4 binding sites (Extended Data Fig. $3 \mathrm{~d} ; P<0.05$

144 by Fisher's exact test, the odds ratio $>1 ; P$-values were corrected by Benjamini-

145 Hochberg procedure). However, they only represented up to $5 \%$ of the maternal TFEs.

146 Therefore, DUX4 does not seem to regulate maternal TFEs (turquoise module) through

147 ERVL-MaLR promoter elements.

To investigate $D U X 4$ functions, we transfected two human embryonic stem cell (hESC)

151 lines H1 and H9 with DUX4-EmGFP TetOn constructs (Fig. 2a and Extended Data Fig.

$1523 \mathrm{a}-\mathrm{d})$ and analysed transcriptome and chromatin status after inducing $D U X 4$ expression

153 using doxicycline. Of the previously reported 32 minor genome activation TFEs ${ }^{8}, 23$

$154(\sim 72 \%)$ including ZSCAN4, TRIM48, LEUTX ${ }^{14}$, and 3 previously unannotated genes

155 (Extended Data Fig. 4) were significantly upregulated in the EmGFP (+) cells (Fig. 2b,

156 Supplementary Information 2). About 74\% (17/23) of the promoters of these TFEs

157 contained DUX4 binding $\operatorname{sites}^{5,13}$ (Fig. 2c). Both, a de novo DNA motif, which was

158 highly similar to the known DUX4 motif, and the known binding site were enriched at

159 the proximal upstream sequence of the upregulated TFEs (Fig. 2d, e). Furthermore, the

160 promoter regions of these transcripts were remarkably overrepresented with DUX4

161 binding sites among hundreds of transcription factors (Extended Data Fig. 3e). In

162 contrast, only $\sim 11 \%(14 / 128)$ of the major embryo genome activation TFEs were up- 
163 regulated (Fig. 2c), suggesting that DUX4 acts only as an inducer of the minor genome

164 activation genes. According to the classification of the differentially regulated TFEs,

165 the vast majority of the upregulated TFEs were mapped to intergenic regions of the

166 genome and the majority of the downregulated TFEs were mapped to the coding

167 regions of the genome (Fig. 2f). We compared these unannotated TFEs upregulated by

168 DUX4 induction with FANTOM-CAT non-coding RNA database and found that 430

169 TFEs overlapped with 394 long non-coding RNA (lncRNA) exons out of which 46

170 were antisense lncRNAs. About 42\% $(1,844 / 4,415)$ of the TFEs overlapped with the

171 ERVL-MaLR elements. Finally, we studied the chromatin status of the EmGFP $(+)$ and

172 EmGFP (-) DUX4 TetOn hESCs using ATAC-sequencing. DUX4 caused rapid

173 chromatin opening (hereafter referred to as ATAC-gained) in the EmGFP $(+)$ cells. Out

174 of these ATAC-gained chromatin sites, 48.9\% significantly overlapped with ERVL-

175 MaLR elements and they were enriched for the DUX4 binding sites $(55.8 \% P<2.2 \mathrm{e}-$

176 16) (Fig. 2g). The ATAC-gained ERVL-MaLR regions remarkably overlapped with the 177 open chromatin regions found in 2-cell human embryos ${ }^{15}$ (Extended Data Fig. 3f). Out

178 of the DUX4-induced gained chromatin regions that overlapped with those of the 2-cell

179 embryos and DUX4 binding sites, $76.7 \%$ were unannotated. This suggests that DUX4

180 is a strong modulator of the intergenic genome immediately after fertilization.

184 Substantial upregulation of intergenic genomic regions after DUX4 induction (Fig. 2f)

185 prompted us to study transcribed enhancers in the DUX4 TetOn hESCs (Fig. 3a). For

186 this, we investigated native elongating transcripts using cap analysis of gene expression 
187 (NET-CAGE) $)^{16}$, which sensitively identifies unstable transcripts such as enhancer

188 RNAs (Extended Data Fig. 5a, b). Integration of our TetOn DUX4 hESC ATAC-seq

189 and NET-CAGE datasets (Fig. 2a and 3a) showed that open chromatin regions were

190 highly enriched at the nucleosome depleted regions of DUX4 induced (Dox+)

191 enhancers but not at enhancers identified in the Dox (-) hESCs (Extended Data Fig. 5c).

192 Altogether, we identified more than 10,000 transcribed enhancers in our Dox $(+)$ DUX4

193 TetOn hESCs and $\sim 90 \%$ of these enhancers have not been identified previously ${ }^{16-18}$

194 (Fig. 3b, Supplementary Information 3). Hereafter, novel enhancers that are exclusive

195 to Dox (+) DUX4 TetOn hESCs are called "novel DUX4 enhancers". Notable, 36.7\%

196 of the novel DUX4 enhancers overlapped with ERVL-MaLR elements (Fig. 3b). We

197 next annotated enhancer expression in our data and identified putative enhancers for

198 three upregulated minor genome activation genes; LEUTX (Fig. 3c, d), previously

199 unannotated RETT FINGER PROTEIN (Fig. 3c, Extended Data Fig. 4), and either for

$200 K H D C 1$ or $K H D C 1 L$ (Fig. 3c, Extended Data Fig. 4). Further, promoters of 12 minor

201 embryo genome activation genes were significantly upregulated by $D U X 4$ induction

202 (Fig. 3c, Supplementary Information 4). We identified the exact promoter position for

203 the ZSCAN4 that is upregulated by DUX4 expression (Extended Data Fig. 5d). We

204 designed guide RNAs for the LEUTX promoter and putative novel enhancer regions

205 (Extended Data Fig. 5e) to experimentally test these using the CRISPRa activation

206 system $^{19}$ in HEK293 cells. The expression level of LEUTX nearly doubled when the

207 guide RNAs targeting the promoter region were transfected together with the putative

208 enhancer 1 targeting guide RNAs in comparison to the promoter targeting guide RNAs

209 only (Fig. 3e). Out of the 56 retroelement families ERVL-MaLRs significantly

210 overlapped with the novel DUX4 enhancers (Fig. 3f and Extended Data Fig. 5f),

211 constituting $\sim 37 \%$ of all novel DUX4 enhancers $(P<2.2 \mathrm{e}-16$; Fig. $3 \mathrm{~b})$. Out of novel 
212 DUX4 enhancers 28\% overlapped with DUX4 binding sites $(P<2.2 \mathrm{e}-16)$. Using DUX4

213 ChIP-seq data we compared whether ERVL-MaLRs regions were more often

214 associated with gene promoter or enhancer regions. Only $9.5 \%$ of the DUX4 binding

215 site overlapping ERVL-MaLRs were associated with promoter regions while $\sim 37 \%$

216 were associated with enhancer regions (Fig. 3g). In summary, DUX4 induces a large

217 number of novel enhancers, many of which overlap with ERVL-MaLR regions and

218 regulate the genome at the time of oocyte-to-embryo transition.

DUX4 protein domains mediating the DUX4 interactions

221 Given predominant DUX4 protein presence in the embryos and stage-specific nuclear

222 localization, we set out to study how DUX4 could mediate such a powerful induction

223 of the genome. For this, we analysed the structural features and protein-protein

224 interactions of DUX4. DUX4 comprises two homeodomains and an intrinsically

225 disordered region with three regions of predicted low disorder conserved in primates.

226 Two predicted amphipathic helices contain a nine amino acid transactivation domain

$227\left(9 \mathrm{aaTAD}{ }^{20}\right)$, also present in $\mathrm{LEUTX}^{21}$, and a motif known to recruit the KIX domain ${ }^{22}$

228 of the cAMP-response element binding protein (CREB)-binding protein (CBP) ${ }^{23}$ (Fig.

229 4a). We modelled the 9aaTAD peptide 371GLLLDELLA379 and the 416EYRALL421

230 peptide (KBM, KIX binding motif) into the MLL and pKID/c-Myb site of the ternary

231 complex NMR structure of human KIX from $\mathrm{CBP}^{24}$ (PDB: 2LXT) (Fig. 4b,

232 Supplementary Information 6). The hydrophobic residues of 9aaTAD and KBM

233 complement well what is seen in the KIX:MLL:pKID complex. Indeed, experimental

234 tight binding (Extended Data Fig. 6 a-c) was detected for peptides overlapping the

$2359 \mathrm{aaTAD}\left(\mathrm{K}_{\mathrm{d}} \approx 0.2 \mu \mathrm{M}\right)$ and $\mathrm{KBM}\left(\mathrm{K}_{\mathrm{d}} \approx 0.6 \mu \mathrm{M}\right)$ sequences of DUX4 to KIX domain, 
and for KBM binding in the presence of 9aaTAD $\left(\mathrm{K}_{\mathrm{d}} \approx 1.1 \mu \mathrm{M}\right)$. Because DUX4 is

237 observed in the cytoplasm (Fig. 1c), we asked whether the homeodomain1-linker-

238 homeodomain2 structure would be stabile as a unit without bound DNA and subjected

239 the crystal structure of DUX4 (PDB: 6E8C ${ }^{25}$, Supplementary Information 7) to

240 molecular dynamics simulations. Ten residues, highly conserved in primates, form two

241 interacting clusters (Extended Data Fig. 6 d, e), stabilizing both domains even in the

242 absence of DNA (Supplementary movie 8). While the predominantly charge-charge

243 interactions hold the two homeodomains together (Extended data Fig. $6 \mathrm{f}-\mathrm{i}$ ), the

244 intermediate linker loop imparts flexibility, which could be vital to accommodate DNA

245 once DUX4 enters the nucleus and locates its binding motif. Indeed, the double

246 homeodomain without DNA opened dramatically, by over $38 \AA$, and the stabile open

247 conformation would be suited to initial interactions with DNA and be consistent with

248 the proposed two-step clamp-like binding mechanism ${ }^{26}$.

To identify protein-protein interactions of DUX4 we used the MAC-tag method where 251 stable (AP-MS) and transient (BioID) interactions can be reliably identified ${ }^{27,28}$. We 252 identified 43 AP-MS and 158 BioID high-confidence DUX4 interactions, out of which 25319 appeared in both datasets (FDR $<0.05$, corresponding to $>0.73$ SAINT Score) 254 (Supplementary Information 9). Overrepresentation Enrichment Analysis (ORA) of 255 protein pathway markers (Reactome, KEGG) showed significant enrichment $(P<0.05$, 256 FDR $<0.01$ ) of markers linked to 'transcription', 'RNA polymerase II Transcription', 257 'chromatin organization' and 'chromatin modifying enzymes'. Comparison of our list 258 of genes to the protein complex databases ComplexPortal and Corum using Fisher's 259 Exact Test yielded significant overrepresentation of the SWI/SNF chromatin 260 remodelling complex, NSL and NuA4 histone acetyltransferase complex, SRCAP 
261 histone exchanging complex, and the Core Mediator complex, $(P<0.05$, FDR $<0.01)$

262 (Fig. 4c). Several DUX4 interacting proteins were classified as RNA binding

263 (GO:0003723), spliceosome and pre-mRNA-splicing (Fig. 4c and SI 10). As the protein

264 interaction assay was performed in the HEK 293 cell line, we studied which of the

265 identified DUX4 interacting proteins are expressed by human oocytes or embryos ${ }^{8,29}$.

266 Nearly all genes coding for the DUX4 interacting proteins were expressed in oocytes,

267 embryos, or both. These results suggested that DUX4 may regulate maternal and

268 embryonic proteins in the cytoplasm and nucleus during the oocyte-to-embryo

269 transition and minor embryonic genome activation.

270

271 


\section{Discussion}

273 The oocyte-to-embryo transition gradually sets the stage for embryo development ${ }^{30-33}$.

274 DUX4 is an obvious primary candidate mediating chromatin and transcriptome changes

275 that are crucial for oocyte-to-embryo transition. Knock-down of DUX4 in the human

276 zygotes did not cause mitotic arrest during the 2-day experiment, in agreement with 277 recent findings on Dux in mouse embryos where a few of the embryos may proceed 278 until the blastocyst stage ${ }^{3,34,35}$. In the mouse Dux-/- embryos, around one third of the 279 embryo genome activation transcripts that are normally upregulated were instead 280 downregulated ${ }^{35}$, while in our human $D U X 4$ knock-down embryos, many of the 281 maternal, normally downregulated genes remained unchanged. This shows that the role 282 of $D U X 4$ in the human is not limited to embryo genome activation but that $D U X 4$ alone 283 is not sufficient for either the oocyte-to-embryo transition or the embryonic genome 284 activation. Given that DUX4 seems to contribute to the maternally biased expressed 285 genes $^{36}$, knockdown at the human zygote stage may hamper observing the entire 286 spectrum of DUX4 functions in the human oocyte-to-embryo transition.

288 Transient upregulation of DUX4 mRNA in zygotes and the increasing nuclear DUX4 289 protein intensity from zygotes until 4-cell stage embryos and its clearance from the 290 nuclei of 8-cell embryos suggested that DUX4 is not only an inducer of the minor 291 embryo genome activation transcripts but that it could also modulate the genome more 292 pervasively and already before genome activation takes place. Ectopic expression of 293 DUX4 in the hESCs caused extensive chromatin opening, largely at newly identified 294 enhancers and ERVL-MaLR elements. Thirty-seven percent of the novel DUX4 295 enhancers were suggested to be derived from ERVL-MaLR elements, indicating that 296 some of the ERVL-MaLRs may function as cells stage specific enhancers in human 
embryos. Indeed, retroelement regions have been suggested to function as regulatory

298 elements, providing novel promoters and possibly enhancers to increase transcriptional

299 complexity especially during development ${ }^{37,38}$. Accordingly, long terminal repeat

300 elements have been suggested as key elements contributing to the oocyte-to-embryo

301 transition $^{39}$. DUX4 upregulation around the time of fertilization may contribute to

302 switching from the maternal ${ }^{40}$ to the cleavage embryo specific retroelements. It is

303 intriguing to speculate, whether activation of ERVL-MaLR elements together with the

304 DUX4 enhancerome provides a correct 'genomic niche' for the subsequent genome

305 activation step. It was recently shown that in mouse Dux binding at Mervl loci drives

306 chromatin reorganisation at these loci in 2-cell embryo-like cell lines, and that

307 chromatin organisation during early mouse development is a consequence of the Mervl

308 integration ${ }^{41}$. To date, human 2-cell-like cell lines have not been established, but

309 importantly, in our experiments, activation of the DUX4 and its likely binding at

310 ERVL-MaLR elements ${ }^{6}$ could modify chromatin towards human cleavage embryo-like

311 stage in the hESCs.

313 According to our DUX4 proteome data some of the strongest DUX4 interactors were

314 the Mediator complex proteins, many of them identified in both stable and transient

315 interactions. Mediator complex proteins interact with both chromatin modifiers and

316 sequence-specific transcription factors ${ }^{42}$ and they have been shown to connect

317 enhancers to promoters ${ }^{43-45}$. The 9 amino acid transactivator domain present in DUX4

318 is known to interact specifically with MED15 transcriptional mediator ${ }^{22}$, also present

319 in our proteome interaction data. Our modelling of the DUX4 protein structure

320 suggested that DUX4 C-terminus contains two transcriptional transactivator domains,

321 the 9aaTAD and the KIX-binding domain. According to our protein model, the KIX 
322 binding motif of the DUX4 appears highly functional, alluding to DUX4 having all the

323 attributes for rapid target binding and activation, as an ideal candidate for rapid

324 modification of a number of genomic regions, including the newly identified enhancers.

325 Therefore, our data suggests that by using its C-terminal domains, DUX4 binds

326 Mediator complex proteins and chromatin modifiers, like $\mathrm{p} 300^{23}$, and modulates the

327 transcriptome, including enhancers, during oocyte-to-embryo transition. In conclusion,

328 DUX4 is a pioneering factor participating in setting the stage for human embryo

329 development. 
Human pre-implantation embryos for single cell RNA-sequencing using single-Cell

335 Tagged Reverse Transcription (STRT)

336 We analysed single cell RNA-sequencing data from Töhönen et al. ${ }^{8}$ for MII oocytes $337(n=20)$, zygotes $(n=59), 2$-cell $(n=4), 4$-cell $(n=15)$ and 8-cell $(n=14)$ embryos. 338 For the DUX4 knockdown experiment, siCTRL cells ( $\mathrm{n}=18$ cells, from two embryos) 339 and siDUX4 cells ( $\mathrm{n}=18$ cells, from three embryos) were analysed. The embryos were 340 incubated in $\mathrm{Ca}^{2+} / \mathrm{Mg}^{2+}$-free culture medium (Biopsy Medium, Origio) at $37^{\circ} \mathrm{C}$ on a

341 heated stage for separation of the cells. Individual cells were briefly rinsed in $342 \mathrm{Ca}^{2+} / \mathrm{Mg}^{2+}$-free PBS and placed directly in lysis buffer $(5 \mathrm{mM}$ Tris- $\mathrm{HCl}, \mathrm{pH} 7.0$ 343 (LifeTechnologies), 5mM DTT (Thermo Scientific), 0.02\% Triton X-100 (Fisher 344 Scientific), $0.5 \mathrm{U} / \mu$ l Ribolock RNAse inhibitor (Thermo Fisher)). The library was 345 prepared according to the published protocol ${ }^{8,46,47}$. The amplified libraries were 346 sequenced on the Illumina HiSeq2000 instrument.

349 TetOn-DUX4 hESCs either with or without doxicycline treatment (see above) were 350 incubated with TrypLE for 5 min, detached, and suspended into cold FACS buffer (5\% 351 FBS in PBS). The cell suspension was filtered through Cell strainers to remove any 352 cell clumps and centrifuged at $107 \mathrm{~g}$ pm for $5 \mathrm{~min}$. The cell pellets from Dox $(+)$ and 353 Dox (-) cultures were suspended in the cold FACS buffer and placed on ice. EmGFP (354 ) cells from the Dox (-) and EmGFP (+) cells from the Dox (+) suspension were sorted 355 into cold FACS buffer using a Sony SH800Z Cell Sorter with blue laser (488) and 100 
$\mu \mathrm{m}$ nozzle. Total RNA was isolated from FAC-sorted DUX4-TetOn hES cells using

357 the RNAqueous Total RNA Isolation Kit (AM1912; Thermo Fisher Scientific). 20 ng

358 of total RNA from each sample was used for library preparations. The libraries were

359 prepared using the STRT method as above, with minor modifications. Briefly, RNA

360 samples were placed in a 48-well plate in which a universal primer, template-switching

361 oligos, and a well-specific 8 bp barcode sequence (for sample identification) were

362 added to each well ${ }^{48,49}$. The synthesized cDNAs from the samples were then pooled

363 into one library and amplified by single-primer PCR with the universal primer

364 sequence. The resulting amplified library was then sequenced using the Illumina

365 NextSeq500 instrument.

368 The sequenced STRT raw reads were processed usin STRTprep ${ }^{48}$ (v3dev branch 369 commit $91 \mathrm{a} 62 \mathrm{~d} 2$ available at https://github.com/shka/STRTprep/tree/v3dev). The 370 processed nonredundant reads were aligned to the hg19 human reference genome 371 sequences. External RNA Control Consortium (ERCC) spike-in sequences and the 372 human ribosomal DNA unit (GenBank: U13369) with RefSeq transcript alignments as 373 a guide of exon junctions. For gene-based statistics, uniquely mapped reads within (i) 374 the 5'-UTR or the proximal upstream (up to $500 \mathrm{bp}$ ) of the RefSeq protein coding genes, 375 and (ii) within the first $50 \mathrm{bp}$ of spike-in sequences, were counted. For TFE-based 376 statistics, the mapped reads were assembled according to the alignments, and uniquely 377 mapped reads within the first exons of the assembled transcripts were counted, as 378 described in Töhönen et al. $2015^{8}$. 
381 Differentially expressed genes and TFEs between two groups had i) significantly

382 different distributions between the two groups by Wilcoxon statistics with multiple 383 resampling ${ }^{49,50}$ (q-value $<0.05$ ), and ii) significantly larger variation than technical 384 variation, which was estimated by variation of the sequenced spike-in RNA levels ${ }^{51,48}$, 385 among all samples of the two groups $(P$-value $<0.05$ adjusted by Benjamini-Hochberg 386 correction). The differential expression was tested by STRTprep pipeline ${ }^{48}$. Enrichment 387 analysis of anatomical terms for the list of upregulated genes by siDUX4 was 388 performed using the TopAnat ${ }^{49}$. All human genes in the Bgee database 389 (https://bgee.org/?page=top_anat $)^{11}$ were used as background. STRT data of the early 390 human embryo were obtained from Töhönen et al. 2015 and $2017^{7,8}$ and were 391 overlapped with TFEs using the intersectBed function from BEDTools ${ }^{52}$ (v2.27.1). 392 DUX4 ChIP-seq data was obtained from GSE33838 and scores around the FEs were 393 calculated with computeMatrix and visualized with plotProfile from deepTools ${ }^{53}$ 394 (v3.1.3). Motif enrichment was analyzed using the command findMotifsGenome.pl 395 from $\mathrm{HOMER}^{54}$ (v4.10.3) with the option “-size -300,100". Enrichment analysis with 396 publicly available ChIP-seq datasets was conducted with ChIP-Atlas ${ }^{55}$ (http://chip397 atlas.org). A total of 7,216 human transcription factor ChIP-seq datasets which had 398 more than 500 peaks were analyzed. Fold enrichment was calculated as (the number of 399 ChIP-seq peaks overlapping with upregulated TFEs / the number of upregulated TFEs) 400 / (the number of ChIP-seq peaks overlapping with all TFEs / the number of all TFEs). $401 P$-values were calculated with Fisher's exact test and $Q$-values were calculated with the 402 Benjamini \& Hochberg method. After excluding the TFEs annotated on ribosomal 403 DNA, 6,425 upregulated TFEs were used as foreground and 109,624 all the detected 404 TFEs were used as background both in the motif and ChIP-seq enrichment analysis. 
407 Nascent RNA from flash-frozen cells was isolated as described by Hirabayashi et al. ${ }^{16}$

408 with the following exceptions: (i) $5 \times$ DNase I enzyme (Thermo Fisher Scientific) was

409 used to prepare the DNase I solution $(50 \mu \mathrm{l})$, (ii) the samples were incubated for up to

$4101 \mathrm{~h}$ at $37^{\circ} \mathrm{C}$ while being pipetted up and down several times every $10 \mathrm{~min}$, and (iii)

411 RNA quality was measured using TapeStation 4200 (Agilent). CAGE-based libraries

412 were generated according to the no-amplification non-tagging CAGE libraries for

413 Illumina next-generation sequencers (nAnT-iCAGE) protocol ${ }^{56}$. All CAGE-based

414 libraries were sequenced in single-read mode on an Illumina NextSeq500 platform.

415 Reads were split by barcode using the MOIRAI ${ }^{57}$ package. Cutadapt $\mathrm{v} 1.1 .8$

416 (http://code.google.com/p/cutadapt/) was used to trim reads to $73 \mathrm{bp}$, and remove reads

417 below base quality 33 and ' $\mathrm{N}$ ' bases. Reads aligning to ribosomal RNA sequences

418 (GenBank U13369.1) were removed using the rRNAdust script within the MOIRAI

419 package. The resulting reads were aligned to the human genome (hg19) using STAR v

$420 \quad 2.5 .0 \mathrm{a}^{58}$ with Gencode v27lift37 ("comprehensive") ${ }^{59}$ as the reference gene model.

421 Mapping was performed with the following parameters: --runThreadN 12 --

422 outSAMtype BAM SortedByCoordinate --out FilterMultimapNmax 1. Following

423 alignment, the technical replicates were merged using the Picard Toolkit v 2.0.1 with

424 the MergeSamFiles program (Broad Institute, Picard Toolkit, 2018.

425 http://broadinstitute.github.io/picard). 
428 Reads mapping to known FANTOM5 promoters $^{60}$ and FANTOM-NET enhancers ${ }^{16}$

429 were counted and normalized essentially as described in $^{16}$. Decomposition peak 430 identification (https://github.com/hkawaji/dpi1/blob/master/identify_tss_peaks.sh)

431 was used to identify tag clusters with default parameters but without decomposition.

432 Peaks with at least three supporting CAGE tags were retained and used as input to

433 identify bidirectional enhancers

434 (https://github.com/anderssonrobin/enhancers/blob/master/scripts/bidir_enhancers).

435 Differential expression (DE) analysis with Benjamini-Hochberg false discovery rate 436 (FDR) correction was performed for promoters and enhancers using egdeR v3.26.8 $8^{61,62}$. 437 Lowly expressed promoters and enhancers (average value between replicates $<-2.5$ $438 \log _{2}$ CPM) were excluded from the analysis. Promoters and enhancers with FDR $\leq 0.01$ 439 were identified as differentially expressed.

442 Putative LEUTX enhancer regions 1 and 2 were predicted from TetOn DUX4 hESC

443 NET-CAGE dataset. The guide RNAs targeting the LEUTX promoter and each of the 444 putative enhancers were designed using the Benchling CRISPR tool 445 (https://benchling.com), targeting them to the proximal promoter ( -400 to -50 base 446 pairs from transcription start site) or the putative enhancers. Possible guide sequences 447 were selected according to their off-target score and position. Guide RNA oligos are 448 shown in Extended Data table 7. Guide RNA transcriptional units (gRNA-PCR) were 449 prepared by PCR amplification and transfected to HEK293 cells as described in Balboa 450 et al. $2015^{63}$. 
453 Guide RNA transcriptional units (gRNA-PCR) were prepared by PCR amplification

454 with Phusion polymerase (Thermo Fisher), using as template U6 promoter and 455 terminator PCR products amplified from $\mathrm{pX} 335$ together with a guide RNA sequence456 containing oligo to bridge the gap. PCR reaction contained 50 pmol forward ( Fw) and 457 reverse (Rev) primers, 2 pmol guide oligo, $5 \mathrm{ng}$ U6 promoter and $5 \mathrm{ng}$ terminator PCR 458 products in a total reaction volume of $100 \mu$. PCR reaction program was $98 \mathrm{C} / 10 \mathrm{sec}$, $45956 \mathrm{C} / 30 \mathrm{sec}, 72 \mathrm{C} / 12 \mathrm{sec}$ for 35 cycles. Amplified gRNA-PCRs were purified. When 460 needed, alternative Fw and Rev primers were used to incorporate suitable restriction 461 sites for gRNAPCR concatenation. LEUTX promoter gRNA-PCR units were 462 concatenated using Golden Gate assembly ${ }^{64}$. Destination vector GGdest-ready was 463 generated by PCR-cloning Esp3I destination cassette from pCAG-T7-TALEN 464 (Sangamo)-Destination (Addgene: $37184^{65}$ into pGEM-4Z (Promega). Assembly 465 reactions contained $150 \mathrm{ng}$ of GGdest-ready vector, $50 \mathrm{ng}$ of each gRNA-PCR product 466 (five in total), $1 \mathrm{uL}$ Esp3I (Thermo Fisher, ER0451), 2 uL T4 DNA ligase (Thermo 467 Fisher, EL0011), 2 uL T4 ligase buffer and 2 uL DTT (10mM, Promega, V3151) in a 468 final volume of $20 \mathrm{uL}$. Thermal cycle consisted of 50 cycles of restriction/ligation (2 $469 \min$ at $37^{\circ} \mathrm{C}, 5 \mathrm{~min}$ at $16^{\circ} \mathrm{C}$ ) followed by enzyme inactivation step $\left(20 \mathrm{~min}\right.$ at $\left.80^{\circ} \mathrm{C}\right)$. 470 Ten microliters of the reaction were transformed into DH5alpha chemical competent 471 bacteria and plated on LB agar containing ampicillin. Correct concatenation of the 472 gRNA-PCR products was confirmed by sequencing. 
475 HEK 293 cells were seeded on tissue culture treated 24 well plates one day prior to

476 transfection $\left(10^{5}\right.$ cells/well). Cells were transfected using FuGENE HD transfection

477 reagent (Promega) in fibroblast culture medium with 500 ng of dCas9VP192

478 transactivator encoding plasmid and $200 \mathrm{ng}$ of gRNA-PCR or gRNA-PCR and vector

479 containing LEUTX promoter targeting guides. Cells were cultured for 72 hours post-

480 transfection, after which samples were collected for qRT-PCR.

Human ESC culture

hESC lines H1 (WA01) and H9 (WA09) were purchased from WiCell. The hESCs were

484 maintained on Geltrex-coated tissue culture dishes in Essential 8 culture medium and 485 passaged every three to five days by incubation with $0.5 \mathrm{mM}$ EDTA (all from Thermo 486 Fisher Scientific).

\section{Plasmid construction}

489 The full-length DUX4 (NM_001293798.2) was synthesized and cloned between the

490 SalI and BamHI sites of the pB-tight-hMAFA-ires-EmGFP-pA-PGK-Puro vector (a 491 kind gift from Diego Balboa, Biomedicum Stem Cell Centre) at GenScript (Genscript, 492 NJ, USA).

495 hESCs were incubated with StemPro Accutase (Thermo Fisher Scientific) until the 496 edges of the colonies started to curl up. The Accutase was aspirated and the cells were 
497

498

499

gently detached in cold 5\% FBS (Thermo Fisher Scientific) $1 \times$ PBS (Corning) and counted. One million cells were centrifuged at $107 \mathrm{~g}$ for $5 \mathrm{~min}$ and the pellet was transferred into $120 \mu \mathrm{l}$ of R-buffer containing $1 \mu \mathrm{g}$ of pB-tight-DUX4-ires-EmGFPpA-PGK-Puro, $0.5 \mu \mathrm{g}$ of pBASE and $0.5 \mu \mathrm{g}$ of rtTA-M2-IN plasmids. $100 \mu \mathrm{l}$ of the cell-plasmid suspension was electroporated with two pulses of $1100 \mathrm{~V}, 20 \mathrm{~ms}$ pulse width, using Neon Transfection system (Thermo Fischer Scientific). The electroporated cells were plated on Geltrex-coated dishes in Essential 8 medium with $10 \mu \mathrm{M}$ ROCK inhibitor Y27632 (Selleckhem). The following day, the medium was exchanged with fresh Essential 8 medium without ROCK inhibitor. The cells were selected with Puromycin at $0.3 \mu \mathrm{g} / \mathrm{ml}$. The TetOn-DUX4 hESC clones were picked manually on Geltrex-coated 96-well plates, expanded and selected again with Puromycin. Appearance of the EmGFP reporter protein was tested using Doxycycline at concentrations ranging from $0.2 \mu \mathrm{g} / \mathrm{ml}$ to $1.0 \mu \mathrm{g} / \mathrm{ml}$ and detected using an EVOS FL Cell imaging system (Thermo Fisher Scientific). For the experiments presented in this paper, the DUX4 TetOn hESCs have been treated with $1 \mu \mathrm{g} / \mathrm{ml}$ of doxycycline for 1,2 , or $3 \mathrm{~h}$ (qPCR) or $4 \mathrm{~h}$ (STRT-RNA seq, ATAC-seq, NET-CAGE) prior to subsequent analyses.

\section{cDNA cloning of previously unannotated genes}

A cDNA library was prepared from a single human 4-cell embryo according to the protocol by Tang et al. ${ }^{66}$ and used for cloning of putative transcripts. Transcripts were amplified using Phusion High-Fidelity DNA polymerase (New England Biolabs) according to manufacturer's instructions. Predicted KHDC1 pseudo gene 1, putative RING-finger type E3 ubiquitin ligase, and putative RING-finger domain protein encoding genes were amplified using touchdown PCR: $98^{\circ} \mathrm{C}$ for $30 \mathrm{~s} ; 24$ cycles of $98^{\circ} \mathrm{C}$ 
522 for $10 \mathrm{~s}$, annealing for $30 \mathrm{~s}$, temperature decreasing from $63^{\circ} \mathrm{C}$ to $56^{\circ} \mathrm{C}, 1^{\circ} \mathrm{C} / 3$ cycles,

$52372^{\circ} \mathrm{C}$ for $30 \mathrm{~s} ; 16$ cycles of $98^{\circ} \mathrm{C}$ for $10 \mathrm{~s}, 55^{\circ} \mathrm{C}$ for $30 \mathrm{~s}, 72^{\circ} \mathrm{C}$ for $30 \mathrm{~s}$; final extension

$52472^{\circ} \mathrm{C}$ for $10 \mathrm{~min}$. All PCR products were cloned into pCR4Blunt-TOPO vector using 525 the Zero Blunt TOPO PCR Cloning kit (Invitrogen) and sequences were verified by 526 Sanger sequencing (Eurofins Genomics). Clone sequences are available from the ENA 527 browser at http://www.ebi.ac.uk/ena/data/view/LR694082-LR694089.

The sequences of the human DUX family proteins were obtained from the UniProt

531 database (The UniProt Consortium), whereas DUX4 sequences from other primates

532 were retrieved from the non-redundant database of NCBI using blastp ${ }^{67}$ with human

533 DUX4 (UniProt ID: Q9UBX2) as the query sequence (SI 5). Multiple sequence

534 alignment over the full-length sequences was carried out using MAFFT ${ }^{68}$ with default 535 parameters. Secondary structures, solvent accessibility and disordered regions were 536 predicted using SCRATCH ${ }^{69}$ and RaptorX-Property ${ }^{70}$. The 9aaTAD web server ("Most 537 Stringent Pattern"71) was used to predict 9aaTAD motifs. The crystal structure of the 538 DUX4 HD1-linker-HD2 fragment bound to DNA (PDB: 6E8C ${ }^{25}$ ) was obtained from 539 the Protein Data Bank (PDB; ${ }^{72}$ ). PyMOL (version 2.4; Schrödinger LLC) and Bodil ${ }^{73}$ 540 were used to analyze inter-HD interactions. For modeling the binding of the 9aaTAD 541 peptide ${ }^{371}$ GLLLDELLA ${ }^{379}$ and the KBM ${ }^{416}$ EYRALL $^{421}$ peptide of DUX4 onto the 542 KIX domain, the NMR structure (model 1/20) of human KIX in complex with MLL 543 and pKID peptide ${ }^{24}$ (PDB: 2LXT) was chosen as the template; the sequence $544{ }^{846}$ PSDIMDFVL ${ }^{854}$ of MLL and ${ }^{13}$ SYRKIL ${ }^{138}$ of pKID were mutated in PyMOL to 545 match the DUX4 sequences ${ }^{371}$ GLLLDELLA ${ }^{379}$ and ${ }^{416}$ EYRALL $^{421}$, respectively, and 
546 the coordinates of extra residues of the MLL and pKID peptides were removed; PDB

547 coordinates for KIX in complex with DUX4 9aaTAD and KBM peptides in 548 Supplementary Information 6.

551 Expression of human KIX domain from CBP, binding of C-terminal peptides

553 A synthetic, codon-optimized gene in pET100/TOPO vector (Invitrogen GeneArt Gene 554 Synthesis, Thermo Scientific) was used to express the human KIX domain of CBP 555 (residues 587-673; Uniprot Q92793) in E. coli BL21 DE3 cells. The expressed 556 construct $(14.5 \mathrm{kDa})$ contained 36 extra $\mathrm{N}$-terminal residues, including a 6xHis tag, the 557 XpressTM epitope and an enterokinase cleavage site, in addition to the KIX domain 558 (86 residues). Transformed E. coli were grown with ampicillin selection in $600 \mathrm{ml}$ of 559 ZYM-5025 autoinduction medium ${ }^{74}$ for $10 \mathrm{~h}$ at $37^{\circ} \mathrm{C}$. The cells were collected by 560 centrifugation at $3,000 \times g$ for $20 \mathrm{~min}$ and stored at $-20^{\circ} \mathrm{C}$. The pellets were thawed and 561 suspended in buffer $\mathrm{A}(50 \mathrm{mM}$ Tris, $\mathrm{pH} 8.0,500 \mathrm{mM} \mathrm{NaCl})$ with $20 \mathrm{mM}$ imidazole and 562 lysed by sonication. The supernatant was separated from the cell debris by 563 centrifugation $(45,000 \times g$ for $40 \mathrm{~min})$ and applied to a three-step purification protocol 564 using an ÄKTA Pure 25 chromatography system (GE) with a UV detector. First, a 565 Histrap HP (1 ml; GE) column was used for metal-affinity chromatography: the sample 566 was applied and the column was subsequently washed with 25 column volumes (CV) 567 of buffer A with $20 \mathrm{mM}$ imidazole. KIX was eluted with a linear imidazole gradient 568 from $20 \mathrm{mM}$ to $500 \mathrm{mM}$ in buffer $\mathrm{A}$ over $15 \mathrm{CV}$, and the column was then washed with $5695 \mathrm{CV}$ of $500 \mathrm{mM}$ imidazole in buffer A. The KIX containing fractions (ca. $7 \mathrm{ml}$ ) were 570 identified by UV absorbance at $280 \mathrm{~nm}$, pooled, then dialyzed (30× volume, two 
exchanges, CelluSep dialysis membrane, MWCO 6-8K; Membrane Filtration Products, Inc.) against buffer $\mathrm{B}(25 \mathrm{mM}$ CHES, pH 9.0). Second, anion exchange chromatography was performed with a Resource Q column (1 ml; GE). The cleared $(3,200 \times g$ for $15 \mathrm{~min})$ dialysis pool was applied to the column, the column was washed with $20 \mathrm{CV}$ of buffer $\mathrm{B}$, and eluted with a linear gradient from 0 to $1 \mathrm{M} \mathrm{NaCl}$ in buffer B over $15 \mathrm{CV}$. The KIX containing fractions were pooled (ca. $4 \mathrm{ml}$ ), then concentrated with an Amicon Ultra-4 centrifugal filter (MWCO 3K; Merck Millipore) to a volume of $0.5 \mathrm{ml}$. Third, the concentrated sample was applied to a Superdex 75 10/300 GL size exclusion chromatography column (GE) and eluted with buffer C (25 mM Tris, pH 8.4, $150 \mathrm{mM} \mathrm{NaCl})$ using a flow rate of $0.5 \mathrm{ml} / \mathrm{min}(0.5 \mathrm{ml}$ fractions $)$. The purity of the sample was analyzed with SDS-PAGE and Coomassie staining, and the concentration was verified by measuring the UV absorbance at $280 \mathrm{~nm}$ with NanoDrop One (Thermo Scientific).

Binding assays were performed using a Monolith NT(TM) microscale thermophoresis instrument (Nanotemper Technologies). The His-tagged KIX domain was labeled noncovalently using Monolith NT(TM) His-Tag Labeling Kit RED-tris-NTA (1st generation; Nanotemper Technologies) according to manufacturer's instructions. Monolith NT.Automated Capillary Chips (Nanotemper Technologies) were used to test binding and to determine the affinity of the 9aaTAD ${ }^{(371}$ GLLLDELLA $\left.^{379}\right)$ and KBM $\left({ }^{416}\right.$ EYRALL $\left.{ }^{421}\right)$ peptides to KIX; the homedomain of human LEUTX with His-Tag was used as a negative control. Peptides were ordered from GenScript and dissolved in deionized water. The final concentration of KIX in the assay was $20 \mathrm{nM}$, and the concentration of each peptide in a binding test assay was $5 \mu \mathrm{M}(250 \times$ molar excess $)$. 
595 The KIX protein and the peptide samples were diluted in PBS-Tween (pH $7.40 .05 \%$

$596 \mathrm{v} / \mathrm{v}$ of Tween 20) buffer for the assays.

Molecular dynamics simulations

599

600 Based on the DUX4 structure (PDB: 6E8C ${ }^{25}$ ), molecular dynamics (MD) simulations,

601 over all atoms, were used to explore the dynamic states of DUX4: (1) double HD 602 complex with (HD1-HD2 + DNA) and (2) without (HD1-HD2) bound DNA, and (3) 603 HD1 + DNA and (4) HD2 + DNA. Prior to the simulations, hydrogen atoms and 604 missing side-chain atoms for R22 of DUX4 were added using Chimera ${ }^{75}$. MD 605 simulations with the AMBER package (version 18; Case, D.A., 2018. The Amber 606 Project, https://ambermd.org/CiteAmber.php) used the ff14SB (for protein; ${ }^{76}$ ) and 607 OL15 (for DNA ${ }^{77}$ ) force fields. The structures were solvated with explicit TIP3P water 608 molecules $^{78}$ within an octahedral box ensuring a $12 \AA$ distance between the boundaries 609 of the simulation box and solute atoms. Sodium counter ions were added to neutralize 610 the system and additional $\mathrm{Na}^{+} / \mathrm{Cl}^{-}$ions were added to bring the salt concentration to 150 $611 \mathrm{mM}$. Periodic boundary conditions were implemented, and the particle-mesh Ewald 612 algorithm was applied ${ }^{79}$ for electrostatic interactions with a distance cutoff of $9 \AA$. For 613 full details of the simulation protocol see Tamirat et al., $2019^{80}$. Conformations were 614 saved every $10 \mathrm{ps}$ and the resulting MD trajectories were analysed further by 615 calculating the root-mean-square deviations (RMSD; over backbone atoms) and root616 mean-square fluctuations (RMSF; over $\mathrm{C} \alpha$ atoms), as well as monitoring hydrogen 617 bond interactions using CPPTRAJ ${ }^{81}$ and $\mathrm{VMD}^{82}$. Coordinates (PDB format) of DUX4 618 HD1-HD2 sans DNA after 100 ns simulation in Supplementary Information 7. 
DUX4 was first amplified in a two-step PCR reaction from pB-tight-DUX4-ires-

622 EmGFP-pA-PGK-Puro and cloned into a Gateway compatible entry clone using

623 Gateway BP Clonase II (Invitrogen) according to manufacturer's instructions. The

624 entry clone was further cloned to Gateway compatible destination vectors containing 625 the C-terminal and N-terminal tags as described ${ }^{28}$. Transfection and selection of the 626 Flp-In ${ }^{\mathrm{TM}}$ T-REx ${ }^{\mathrm{TM}} 293$ cells (Invitrogen, Life Technologies, R78007, cultured in 627 manufacturer's recommended conditions) and affinity purification of the final product was done as previously ${ }^{28}$.

\section{Liquid Chromatography-Mass Spectrometry}

Analysis was performed on a Q-Exactive mass spectrometer with an EASY-nLC 1000 system via an electrospray ionization sprayer (Thermo Fisher Scientific), using Xcalibur version 3.0.63. Peptides were eluted from the sample with a C18 precolumn

634 (Acclaim PepMap 100, $75 \mu \mathrm{m} \times 2 \mathrm{~cm}, 3 \mu \mathrm{m}, 100 \AA \AA$; Thermo Scientific) and analytical 635 column (Acclaim PepMap RSLC, $65 \mu \mathrm{m} \times 15 \mathrm{~cm}, 2 \mu \mathrm{m}, 100 \AA$; Thermo Scientific), 636 using a 60 minute buffer gradient ranging from $5 \%$ to $35 \%$ Buffer $\mathrm{B}$, then a 5 min 637 gradient from $35 \%$ to $80 \%$ Buffer B and 10 minute gradient from $80 \%$ to $100 \%$ Buffer 638 B ( $0.1 \%$ formic acid in $98 \%$ acetonitrile and $2 \%$ HPLC grade water $) .4 \mu 1$ of peptide 639 sample was loaded by a cooled autosampler. Data-dependent FTMS acquisition was in 640 positive ion mode for $80 \mathrm{~min}$. A full scan $(200-2000 \mathrm{~m} / \mathrm{z})$ was performed with a 641 resolution of 70,000 followed by top $10 \mathrm{CID}-\mathrm{MS}^{2}$ ion trap scans with a resolution of 642 17,500. Dynamic exclusion was set for 30 s. Database search was performed with 643 Proteome Discoverer 1.4 (Thermo Scientific) using the SEQUEST search engine on the 
644 Reviewed human proteome in UniProtKB/SwissProt databases

645 (http://www.uniprot.org, downloaded Nov. 2018). Trypsin was selected as the cleavage

646 enzyme and maximum of 2 missed cleavages were permitted, precursor mass tolerance

647 at \pm 15 ppm and fragment mass tolerance at $0.05 \mathrm{Da}$. Carbamidomethylation of cysteine

648 was defined as a static modification. Oxidation of methionine and biotinylation of

649 lysine and N-termini were set as variable modifications. All reported data were based

650 on high-confidence peptides assigned in Proteome Discoverer (FDR $<0.05$ ).

651

652 Identification of statistical confidence of interactions

653 Significance Analysis of INTeractome (SAINT ${ }^{83}$ )-express version 3.6 .3 and

654 Contaminant Repository for Affinity Purification (CRAPome,

655 http://www.crapome.org) were used to discover statistically significant interactions

656 from the AP-MS data ${ }^{84}$. The DUX4 LC-MS data was ran alongside a large dataset of

657 other transcription factors, as well as a large GFP control set. Final results represent

658 proteins with a SAINT score higher than 0.73 , and present in all four replicates.

659

660 Overrepresentation Analysis

661 Overrepresentation analysis of statistically significant interactions in Gene Ontology

662 and Reactome was done in WebGestalt ${ }^{85}$, and overrepresentation of prey proteins in

663 ComplexPortal ${ }^{86}$ (https://www.ebi.ac.uk/complexportal) and CORUM $^{87}$

664 (https://mips.helmholtz-muenchen.de/corum/) was done using Fisher's exact test and

665 multiple testing correction in an in-house R-script. 
668 Protein interaction networks were constructed from filtered SAINT data that was

669 imported to Cytoscape 3.6.0. Known prey-prey interactions were obtained from the 670 iRef database (http://irefindex.org).

671

$R N A$ isolation, reverse transcription and quantitative real-time PCR from DUX4 TetOn

673 hESCS

Total RNA was isolated using NucleoSpin RNA kit (Macherey Nagel). $1 \mu \mathrm{g}$ of RNA was reverse transcribed by MMLV-RTase with oligo dT, dNTPs, and Ribolock in MMLV-RTase buffer (Thermo Fisher Scientific). 5× HOT FIREPol qPCR Mix (Solis

677 Biodyne) was used to measure relative mRNA levels with LightCycler (Roche). The $678 \Delta \Delta \mathrm{CT}$ method was followed to quantify the relative gene expression where 679 CYCLOPHILIN $G$ was used as endogenous control. Relative expression of each gene 680 was normalized to the expression without doxicycline treatment. The primer sequences 681 are listed in Extended Data Table 7.

682

684 The ATAC-sequencing libraries were prepared as in ${ }^{88} .5 \times 10^{4}$ EmGFP (-) and EmGFP 685 (+) TetOn-hESCs (H1 clone 2, H1 clone 8, H9 clone 3 and H9 clone 4) were centrifuged 686 at $500 \times g$ for $5 \mathrm{~min}$. The pellets were washed in cold $1 \times \mathrm{PBS}$ by centrifugation at $500 \times g$ 687 for $5 \mathrm{~min}$. Each cell pellet was lysed in $50 \mu \mathrm{l}$ of cold lysis buffer (10 mM Tris-HCl, pH 7.4; $10 \mathrm{mM} \mathrm{NaCl}, 3 \mathrm{mM} \mathrm{MgCl}$, and 0.1\% IGEPAL CA-630) and centrifuged at $500 \times g$ at $4^{\circ} \mathrm{C}$ for $10 \mathrm{~min}$. The pellet was then resuspended in the transposase reaction mix $(2.5$ 
$690 \mu 1$ of transposase in TD buffer (Nextera DNA library preparation kit, Illumina) and

691 incubated at $37^{\circ} \mathrm{C}$ for $30 \mathrm{~min}$. The reactions were purified through columns and eluted

692 in $20 \mu 1$. After addition of the barcode oligos the DNA samples were amplified for 12 693 cycles $\left(98^{\circ} \mathrm{C}\right.$ for $10 \mathrm{~s}, 63^{\circ} \mathrm{C}$ for $30 \mathrm{~s}$ and $72^{\circ} \mathrm{C}$ for $\left.60 \mathrm{~s}\right)$ in Phusion PCR master mix 694 (Thermo Fisher Scientific). The PCR products were purified through the columns and 695 eluted in $20 \mu 1$.

696 ATAC-seq data analysis

698 Bcl files were converted and demultiplexed to fastq using the bcl2fastq program. 699 STAR $^{58}$ was used to index the human reference genome (hg19), obtained from UCSC, 700 and align the resulting fastq files. The resulting bam files with the mapped reads were 701 then converted to tag directories with subsequent peaks calling using the HOMER suit 702 of programs ${ }^{54}$. HOMER was also employed for counting the reads in the identified peak 703 regions. The raw tag counts from the peaks were then imported to R/Bioconductor and 704 differential peak analysis was performed using the edgeR package and its general linear 705 models pipeline. Peaks with an FDR adjusted $\mathrm{p}$ value under 0.05 were termed 706 significant. Plotting was done in $\mathrm{R}$ using packages Complex heatmap, ggplot2 and 707 ggbeeswarm. RepeatMasker table downloaded from UCSC 708 (http://hgdownload.soe.ucsc.edu/goldenPath/hg19/database/rmsk.txt.gz) was 709 converted to BED format and then intersected with the ATAC-seq peaks using the 710 intersectBed from BEDTools ${ }^{52}$ to determine the peaks overlapped with ERVL-MaLR 711 elements. ATAC-seq data of human early embryo were obtained from GSE101571 15 , 712 and scores around the ATAC-seq peaks were calculated with computeMatrix and 
713 visualized with plotHeatmap from deepTools ${ }^{53}$. All the scripts and command line

714 options can be provided upon request.

717 Cells were fixed with $3.8 \%$ PFA, washed three times, permeabilised in $0.5 \%(\mathrm{v} / \mathrm{v})$

718 Triton X-100 in PBS for 7 min, and washed with washing buffer $(0.1 \%(\mathrm{v} / \mathrm{v})$ Tween20

719 in PBS). The samples were incubated with ProteinBlock (Thermo Fisher Scientific) at

720 room temperature for $10 \mathrm{~min}$ to prevent unspecific binding of primary antibody.

721 Primary antibody (rabbit MAb anti-DUX4, clone E5-5, Abcam) was diluted 1:300 in

722 washing buffer and incubated at $4^{\circ} \mathrm{C}$ overnight. After washings, fluorescence723 conjugated secondary antibody (anti rabbit 594, A-21207; Thermo Fisher Scientific) 724 was diluted 1:1000 in washing buffer and incubated at room temperature for $20 \mathrm{~min}$.

725 Nuclei were counterstained with DAPI 1:1000 in washing buffer. The images were 726 captured with an Evos FL Cell Imaging system using 10× and 20× Plan Achromatic 727 objectives.

730 For characterization and quantitation of the DUX4 protein zygotes $(n=3)$ and embryos

731 (2-cell, $\mathrm{n}=3 ; 4$-cell, $\mathrm{n}=4 ; 8$-cell, $\mathrm{n}=2$ plus one early 8 -cell stage embryo shown in

732 Extended Data Fig. 1 were fixed in $3.8 \%$ PFA at room temperature for 15 min, washed

733 three times in washing buffer (as above), and permeabilised in 0.5\% Triton X-100 in

734 PBS at room temperature for $15 \mathrm{~min}$. Unspecific primary antibody binding was blocked 735 as above. DUX4 antibody (as above) was incubated at $4{ }^{\circ} \mathrm{C}$ overnight. The embryos 
736

737 Thermo Fisher Scientific) diluted 1:500 in washing buffer (as above) at room

738 temperature for $2 \mathrm{~h}$. After washings, nuclei were counterstained with DAPI 1:500 in

739 washing buffer. To confirm that DUX4 targeting siRNA efficiently downregulated

740 DUX4, siCTRL $(\mathrm{n}=4)$ and siDUX4 $(\mathrm{n}=5)$ microinjected zygotes were stained for DUX4

741 as above.

742

743 Imaging and confocal microscopy image analysis

744 Human embryos were imaged in washing buffer on Ibidi 8-well $\mu$ slides with a Leica

745 TCS SP8 confocal laser scanning microscope (Leica Microsystems, Mannheim,

746 Germany) using Leica HC PL APO CS2 40×/1.10NA and Leica HC PL APO CS2

$74763 \times / 1.20 \mathrm{NA}$ water objectives. Confocal images were processed using Fiji

748 (http://fiji.sc). For the data presented in Fig 1c and d, images were smoothened using a

749 Gaussian filter (radius $=1$ pixel kernel). For the quantification of the DUX4 intensity

750 in the nucleus (Fig. 1d), the DAPI channel was denoised using a rolling ball (radius =

751 100). The images were smoothened in 3D using a Gaussian filter (radius $=2$ pixel

752 kernel) and cell nuclei were segmented. The segmented regions were used to measure

753 average pixel intensity per nucleus in each cell in the DUX4 channel. DUX4 intensity

754 in the nucleus was normalized to intensity of the corresponding cytoplasmic DUX4

755 staining in the single representative plane. 
758 Human triploid zygotes were warmed using a Gems Warming Set (Genea Biomedx) 759 and cultured in G-TL medium (Vitrolife) in $6 \% \mathrm{O}_{2}$ and $6 \% \mathrm{CO}_{2}$ at $37^{\circ} \mathrm{C} .12 \mu \mathrm{l}$ of either

$76020 \mu \mathrm{M}$ scrambled control siRNA (AM4611, Thermo Fisher Scientific) or DUX4761 targeting siRNA (cat. 4457308, Thermo Fisher Scientific) diluted in nucleotide-free $762 \mathrm{H}_{2} \mathrm{O}$ were mixed with total of $500 \mathrm{ng}$ of GAP-GFP mRNA and centrifuged at maximum 763 speed at $4^{\circ} \mathrm{C}$ for $10 \mathrm{~min}$. The embryos were microinjected using Eppendorf 764 microinjector and placed in G-TL medium in a Geri dish for 3D time-lapse imaging 765 (Geri incubator, Genea Biomedx, Australia).

Human embryo live imaging

768 Imaging of the human triploid embryos was initiated immediately after microinjections 769 (Geri incubator). Images were captured in 3D every 15 min until the embryos were removed for fluorescence staining or termination of the experiment.

\section{Ethical approvals}

773 Collection and experiments on human oocytes and embryos were approved by the

774 Helsinki University Hospital ethical committee, diary numbers 308/13/03/03/2015 and

775 HUS/1069/2016. Human surplus oocytes, zygotes, and embryos were donated by 776 couples that had undergone infertility treatments at the Reproduction Medicine Unit of 777 the Helsinki University Hospital. The donations were done with an informed consent 778 and patients understood that donating oocytes, zygotes, or embryos is voluntary and 779 does not affect their treatment in any way. 
782 We are grateful for all the couples that donated their surplus oocytes, zygotes, or 783 embryos for this project. We thank the IVF nurses at the Reproductive Medicine Unit 784 of the Helsinki University Hospital for recruiting the couples for the oocyte and embryo 785 donation program, Dr. Diego Balboa-Alonso for the pB-ires-EmGFP-pA-PGK-Puro 786 plasmid and Dr. Jere Weltner for insightful discussions. We acknowledge Biomedicum 787 Imaging Unit (Helsinki), Functional Genomics Unit (Helsinki), Biomedicum Flow 788 Cytometry Unit (Helsinki), Bioinformatics and Expression Analysis Core Facility 789 (Stockholm) for skilled technical assistance. We thank Dr. Jukka Lehtonen for 790 scientific IT support (Biocenter Finland) and CSC IT Center for Science for 791 supercomputing. We thank prof. Outi Hovatta for inspiration and introducing the senior 792 author to the field of early embryogenesis. This project was supported by Jane and 793 Aatos Erkko foundation, Sigrid Jusélius foundation, Finnish Academy, and Helsinki 794 University Hospital funds. SV was supported by Jane and Aatos Erkko foundation. MY 795 was supported by the Scandinavia-Japan Sasakawa Foundation, the Japan Eye Bank 796 Association, the Astellas Foundation for Research on Metabolic Disorders, and the 797 Japan Society for the Promotion of Science Overseas Research Fellowships. MT was 798 supported by Joe, Pentti and Tor Memorial Foundation, Graduate School of Åbo 799 Akademi University. VR was supported by Foundation of Åbo Akademi University 800 and Magnus Ehrnrooth Foundation. 
803 SV, SK and JK conceived and coordinated the study. YM, TRB, MV, MSJ, TT, SK

804 and JK supervised the work in each contributing laboratory. Every author participated

805 in either planning or conducting respective experiments and analysing or interpreting

806 the data. SV, MY, VR, TA, MT, MSJ, JK wrote the manuscript. All authors approved

807 the final version of the manuscript.

808

809

Competing interests

810 The authors declare no competing interests.

813 This manuscript contains Supplementary Information.

Supplementary Information 1. Supplementary file showing expression levels and

blastomeres. The descriptions of the columns are available at:

818 https://github.com/shka/STRTprep/blob/v3dev/doc/result.md\#outbygenediffexpxls

820 Supplementary Information 2. Supplementary file showing expression levels and

822 samples. The descriptions of the columns are available at:

823 https://github.com/shka/STRTprep/blob/v3dev/doc/result.md\#outbygenediffexpxls

825 Supplementary Information 3. Supplementary table showing significantly

826 differentially expressed enhancers in Dox (+) versus Dox (-) DUX4 TetOn hESCs. 
828 Supplementary Information 4. Supplementary table showing significantly

829 differentially expressed promoters in Dox (+) versus Dox (-) DUX4 TetOn hESCs.

831 Supplementary Information 5. Supplementary table showing DUX family proteins

832 in primates.

833

834 Supplementary information 6. Supplementary table showing coordinates (KIX-

835 9aaTAD-KBM.pdb) for modeled KIX in complex with DUX4 9aaTAD and KBM 836 peptides.

837

838 Supplementary information 7. Supplementary table showing coordinates

839 (DUX4_HD1-HD2.pdb) of DUX4 HD1-HD2 without bound DNA at the end of a 100

840 ns molecular dynamics simulation.

842 Supplementary information 8. Two concatenated supplementary movies.

843 First, $360^{\circ}$ view of last sampled conformation of DNA-free DUX4 (blue) from the

844100 ns simulation superposed on the DNA-bound DUX4 crystal structure (red and

845 grey) (HD1-HD2-comparison.mp4). Second, molecular dynamics simulation (100 ns)

846 of DUX4 HD1-HD2 without bound DNA (HD1-HD2.mp4).

848 Supplementary Information 9. Supplementary table showing transient (BioID) and 849 stable (AP-MS) DUX4 protein - protein interactions as well as interactions found by 850 both BioID and AP-MS methods (both). 
8531 Jukam, D., Shariati, S. A. M. \& Skotheim, J. M. Zygotic Genome Activation

$854 \quad$ in Vertebrates. Developmental cell 42, 316-332,

855 doi:10.1016/j.devcel.2017.07.026 (2017).

8562 Conti, M. \& Franciosi, F. Acquisition of oocyte competence to develop as an

857 embryo: integrated nuclear and cytoplasmic events. Human reproduction

858 update 24, 245-266, doi:10.1093/humupd/dmx040 (2018).

8593 De Iaco, A. et al. DUX-family transcription factors regulate zygotic genome $860 \quad$ activation in placental mammals. Nat Genet 49, 941-945, doi:10.1038/ng.3858 (2017).

8624 Hendrickson, P. G. et al. Conserved roles of mouse DUX and human DUX4 in activating cleavage-stage genes and MERVL/HERVL retrotransposons.

864 Nature genetics 49, 925-934, doi:10.1038/ng.3844 (2017).

5 Geng, L. N. et al. DUX4 activates germline genes, retroelements, and immune mediators: implications for facioscapulohumeral dystrophy. Developmental cell 22, 38-51, doi:10.1016/j.devcel.2011.11.013 (2012).

869

6 Whiddon, J. L., Langford, A. T., Wong, C. J., Zhong, J. W. \& Tapscott, S. J. Conservation and innovation in the DUX4-family gene network. Nat Genet 49, 935-940, doi:10.1038/ng.3846 (2017).

Tohonen, V. et al. Transcriptional activation of early human development suggests DUX4 as an embryonic regulator. doi:https://doi.org/10.1101/123208 (2017).

8 Tohonen, V. et al. Novel PRD-like homeodomain transcription factors and retrotransposon elements in early human development. Nature communications 6, 8207, doi:10.1038/ncomms9207 (2015). growth/differentiation factor-9. Molecular endocrinology 9, 131-136, doi:10.1210/mend.9.1.7760846 (1995).

10 Canosa, S. et al. Zona pellucida gene mRNA expression in human oocytes is related to oocyte maturity, zona inner layer retardance and fertilization competence. Molecular human reproduction 23, 292-303, doi:10.1093/molehr/gax008 (2017). B. BgeeDB, an R package for retrieval of curated expression datasets and for gene list expression localization enrichment tests. F1000Research 5, 2748, doi:10.12688/f1000research.9973.2 (2016). network analysis. BMC bioinformatics 9, 559, doi:10.1186/1471-2105-9-559 (2008). 
13 Young, J. M. et al. DUX4 binding to retroelements creates promoters that are active in FSHD muscle and testis. PLoS genetics 9, e1003947, doi:10.1371/journal.pgen.1003947 (2013).

14 Jouhilahti, E. M. et al. The human PRD-like homeobox gene LEUTX has a central role in embryo genome activation. Development 143, 3459-3469, doi:10.1242/dev.134510 (2016).

$15 \mathrm{Wu}$, J. et al. Chromatin analysis in human early development reveals epigenetic transition during ZGA. Nature 557, 256-260, doi:10.1038/s41586018-0080-8 (2018).

16 Hirabayashi, S. et al. NET-CAGE characterizes the dynamics and topology of human transcribed cis-regulatory elements. Nat Genet 51, 1369-1379, doi:10.1038/s41588-019-0485-9 (2019).

17 Andersson, R. et al. An atlas of active enhancers across human cell types and tissues. Nature 507, 455-461, doi:10.1038/nature12787 (2014).

18 Arner, E. et al. Transcribed enhancers lead waves of coordinated transcription in transitioning mammalian cells. Science 347, 1010-1014, doi:10.1126/science.1259418 (2015).

19 Weltner, J. et al. Human pluripotent reprogramming with CRISPR activators. Nature communications 9, 2643, doi:10.1038/s41467-018-05067-x (2018).

20 Mitsuhashi, H. et al. Functional domains of the FSHD-associated DUX4 protein. Biology open 7, doi:10.1242/bio.033977 (2018).

21 Katayama, S. et al. Phylogenetic and mutational analyses of human LEUTX, a homeobox gene implicated in embryogenesis. Scientific reports $\mathbf{8}, 17421$, doi:10.1038/s41598-018-35547-5 (2018).

22 Piskacek, M., Havelka, M., Rezacova, M. \& Knight, A. The 9aaTAD Transactivation Domains: From Gal4 to p53. PloS one 11, e0162842, doi:10.1371/journal.pone.0162842 (2016).

23 Choi, S. H. et al. DUX4 recruits p300/CBP through its C-terminus and induces global H3K27 acetylation changes. Nucleic acids research 44, 51615173, doi:10.1093/nar/gkw141 (2016).

24 Bruschweiler, S., Konrat, R. \& Tollinger, M. Allosteric communication in the KIX domain proceeds through dynamic repacking of the hydrophobic core. ACS chemical biology 8, 1600-1610, doi:10.1021/cb4002188 (2013).

25 Lee, J. K. et al. Crystal Structure of the Double Homeodomain of DUX4 in Complex with DNA. Cell reports 25, 2955-2962 e2953, doi:10.1016/j.celrep.2018.11.060 (2018).

26 Dong, X. et al. Structural basis of DUX4/IGH-driven transactivation. Leukemia 32, 1466-1476, doi:10.1038/s41375-018-0093-1 (2018).

27 Varjosalo, M. et al. Interlaboratory reproducibility of large-scale human protein-complex analysis by standardized AP-MS. Nature methods 10, 307314, doi:10.1038/nmeth.2400 (2013).

28 Liu, X. et al. An AP-MS- and BioID-compatible MAC-tag enables comprehensive mapping of protein interactions and subcellular localizations. Nature communications 9, 1188, doi:10.1038/s41467-018-03523-2 (2018). 
29 Yan, L. et al. Single-cell RNA-Seq profiling of human preimplantation embryos and embryonic stem cells. Nature structural \& molecular biology 20, 1131-1139, doi:10.1038/nsmb.2660 (2013).

30 Tadros, W. \& Lipshitz, H. D. The maternal-to-zygotic transition: a play in two acts. Development 136, 3033-3042, doi:10.1242/dev.033183 (2009).

31 Walser, C. B. \& Lipshitz, H. D. Transcript clearance during the maternal-tozygotic transition. Current opinion in genetics \& development 21, 431-443, doi:10.1016/j.gde.2011.03.003 (2011).

32 Lee, M. T., Bonneau, A. R. \& Giraldez, A. J. Zygotic genome activation during the maternal-to-zygotic transition. Annual review of cell and developmental biology 30, 581-613, doi:10.1146/annurev-cellbio-100913013027 (2014).

33 Schultz, R. M., Stein, P. \& Svoboda, P. The oocyte-to-embryo transition in mouse: past, present, and future. Biology of reproduction 99, 160-174, doi:10.1093/biolre/ioy013 (2018).

34 Chen, Z. \& Zhang, Y. Loss of DUX causes minor defects in zygotic genome activation and is compatible with mouse development. Nat Genet 51, 947-951, doi:10.1038/s41588-019-0418-7 (2019).

35 De Iaco, A., Verp S., Offner S., Trono D. DUX is a non-essential synchronizer of zygotic genome activation. doi:https://doi.org/10.1101/569434 (2019).

36 Leng, L. et al. Single-Cell Transcriptome Analysis of Uniparental Embryos Reveals Parent-of-Origin Effects on Human Preimplantation Development. Cell stem cell 25, 697-712 e696, doi:10.1016/j.stem.2019.09.004 (2019).

37 Thompson, P. J., Macfarlan, T. S. \& Lorincz, M. C. Long Terminal Repeats: From Parasitic Elements to Building Blocks of the Transcriptional Regulatory Repertoire. Molecular cell 62, 766-776, doi:10.1016/j.molcel.2016.03.029 (2016).

38 Nishihara, H. et al. Coordinately Co-opted Multiple Transposable Elements Constitute an Enhancer for wnt5a Expression in the Mammalian Secondary Palate. PLoS genetics 12, e1006380, doi:10.1371/journal.pgen.1006380 (2016).

39 Franke, V. et al. Long terminal repeats power evolution of genes and gene expression programs in mammalian oocytes and zygotes. Genome research 27, 1384-1394, doi:10.1101/gr.216150.116 (2017).

40 Georgiou, I. et al. Retrotransposon RNA expression and evidence for retrotransposition events in human oocytes. Human molecular genetics $\mathbf{1 8}$, 1221-1228, doi:10.1093/hmg/ddp022 (2009).

41 Kruse K., D. N., Enriquez-Gasca R., Gaume X., Torres-Padilla M-E., Vaquerizas JM. Transposable elements drive reorganisation of 3D chromatin during early embryogenesis. doi:https://doi.org/10.1101/523712 (2019).

42 Meng, H. \& Bartholomew, B. Emerging roles of transcriptional enhancers in chromatin looping and promoter-proximal pausing of RNA polymerase II. The Journal of biological chemistry 293, 13786-13794, doi:10.1074/jbc.R117.813485 (2018). 
43 Quevedo, M. et al. Mediator complex interaction partners organize the transcriptional network that defines neural stem cells. Nature communications 10, 2669, doi:10.1038/s41467-019-10502-8 (2019).

44 Petrenko, N., Jin, Y., Wong, K. H. \& Struhl, K. Mediator Undergoes a Compositional Change during Transcriptional Activation. Molecular cell 64, 443-454, doi:10.1016/j.molcel.2016.09.015 (2016).

45 Jeronimo, C. et al. Tail and Kinase Modules Differently Regulate Core Mediator Recruitment and Function In Vivo. Molecular cell 64, 455-466, doi:10.1016/j.molcel.2016.09.002 (2016).

46 Islam, S. et al. Highly multiplexed and strand-specific single-cell RNA 5' end sequencing. Nature protocols 7, 813-828, doi:10.1038/nprot.2012.022 (2012).

47 Islam, S. et al. Quantitative single-cell RNA-seq with unique molecular identifiers. Nature methods 11, 163-166, doi:10.1038/nmeth.2772 (2014).

48 Krjutskov, K. et al. Single-cell transcriptome analysis of endometrial tissue. Human reproduction 31, 844-853, doi:10.1093/humrep/dew008 (2016).

49 Katayama, S., Tohonen, V., Linnarsson, S. \& Kere, J. SAMstrt: statistical test for differential expression in single-cell transcriptome with spike-in normalization. Bioinformatics 29, 2943-2945, doi:10.1093/bioinformatics/btt511 (2013).

50 Li, J. \& Tibshirani, R. Finding consistent patterns: a nonparametric approach for identifying differential expression in RNA-Seq data. Statistical methods in medical research 22, 519-536, doi:10.1177/0962280211428386 (2013).

51 Brennecke, P. et al. Accounting for technical noise in single-cell RNA-seq experiments. Nature methods 10, 1093-1095, doi:10.1038/nmeth.2645 (2013).

52 Quinlan, A. R. \& Hall, I. M. BEDTools: a flexible suite of utilities for comparing genomic features. Bioinformatics 26, 841-842, doi:10.1093/bioinformatics/btq033 (2010).

53 Ramirez, F., Dundar, F., Diehl, S., Gruning, B. A. \& Manke, T. deepTools: a flexible platform for exploring deep-sequencing data. Nucleic acids research 42, W187-191, doi:10.1093/nar/gku365 (2014).

54 Heinz, S. et al. Simple combinations of lineage-determining transcription factors prime cis-regulatory elements required for macrophage and B cell identities. Molecular cell 38, 576-589, doi:10.1016/j.molcel.2010.05.004 (2010).

55 Oki, S. et al. ChIP-Atlas: a data-mining suite powered by full integration of public ChIP-seq data. EMBO reports 19, doi:10.15252/embr.201846255 (2018).

Murata, M. et al. Detecting expressed genes using CAGE. Methods in molecular biology 1164, 67-85, doi:10.1007/978-1-4939-0805-9_7 (2014).

57 Hasegawa, A., Daub, C., Carninci, P., Hayashizaki, Y. \& Lassmann, T. MOIRAI: a compact workflow system for CAGE analysis. $B M C$ bioinformatics 15, 144, doi:10.1186/1471-2105-15-144 (2014). 29, 15-21, doi:10.1093/bioinformatics/bts635 (2013). 
102359 Harrow, J. et al. GENCODE: the reference human genome annotation for The

1024

1025

1026

1027

1028

1029

1030

1031

1032

1033

1034

1035

1036

1037

1038

1039

1040

1041

1042

$1043 \quad 66$

1044

1045

1046

1047

1048

1049

1050

1051

1052

1053

1054

1055

1056

1057

1058

1059

1060

1061

1062

1063

1064

1065

1066

ENCODE Project. Genome research 22, 1760-1774, doi:10.1101/gr.135350.111 (2012).

60 Consortium, F. et al. A promoter-level mammalian expression atlas. Nature 507, 462-470, doi:10.1038/nature13182 (2014).

61 Robinson, M. D., McCarthy, D. J. \& Smyth, G. K. edgeR: a Bioconductor package for differential expression analysis of digital gene expression data. Bioinformatics 26, 139-140, doi:10.1093/bioinformatics/btp616 (2010).

62 McCarthy, D. J., Chen, Y. \& Smyth, G. K. Differential expression analysis of multifactor RNA-Seq experiments with respect to biological variation. Nucleic acids research 40, 4288-4297, doi:10.1093/nar/gks042 (2012).

63 Balboa, D. et al. Conditionally Stabilized dCas9 Activator for Controlling Gene Expression in Human Cell Reprogramming and Differentiation. Stem cell reports 5, 448-459, doi:10.1016/j.stemcr.2015.08.001 (2015).

64 Cermak, T. et al. Efficient design and assembly of custom TALEN and other TAL effector-based constructs for DNA targeting. Nucleic acids research 39, e82, doi:10.1093/nar/gkr218 (2011).

65 Hermann, M., Cermak, T., Voytas, D. F. \& Pelczar, P. Mouse genome engineering using designer nucleases. Journal of visualized experiments : JoVE, doi:10.3791/50930 (2014).

66 Tang, F. et al. RNA-Seq analysis to capture the transcriptome landscape of a single cell. Nature protocols 5, 516-535, doi:10.1038/nprot.2009.236 (2010).

67 Johnson, M. et al. NCBI BLAST: a better web interface. Nucleic acids research 36, W5-9, doi:10.1093/nar/gkn201 (2008).

68 Katoh, K. \& Standley, D. M. MAFFT multiple sequence alignment software version 7: improvements in performance and usability. Molecular biology and evolution 30, 772-780, doi:10.1093/molbev/mst010 (2013).

69 Cheng, J., Randall, A. Z., Sweredoski, M. J. \& Baldi, P. SCRATCH: a protein structure and structural feature prediction server. Nucleic acids research 33, W72-76, doi:10.1093/nar/gki396 (2005).

70 Wang, S., Li, W., Liu, S. \& Xu, J. RaptorX-Property: a web server for protein structure property prediction. Nucleic acids research $44, \mathrm{~W} 430-435$, doi:10.1093/nar/gkw306 (2016).

71 Piskacek, S. et al. Nine-amino-acid transactivation domain: establishment and prediction utilities. Genomics 89, 756-768, doi:10.1016/j.ygeno.2007.02.003 (2007).

72 Berman, H. M. et al. The Protein Data Bank. Nucleic acids research 28, 235242, doi:10.1093/nar/28.1.235 (2000).

73 Lehtonen, J. V. et al. BODIL: a molecular modeling environment for structure-function analysis and drug design. Journal of computer-aided molecular design 18, 401-419, doi:10.1007/s10822-004-3752-4 (2004).

74 Studier, F. W. Protein production by auto-induction in high density shaking cultures. Protein expression and purification 41, 207-234, doi:10.1016/j.pep.2005.01.016 (2005). 
$106775 \quad$ Pettersen, E. F. et al. UCSF Chimera--a visualization system for exploratory

1068

1069

1070

1071

1072

1073

1074

1075

1076

1077

1078

1079

1080

1081

1082

1083

1084

1085

1086

1087

1088

1089

1090

1091

1092

1093

1094

1095

1096

1097

1098

1099

1100

1101

$1102 \quad 86$

1103

1104

1105

1106

1107

$1108 \quad 88$

1109 research and analysis. Journal of computational chemistry 25, 1605-1612, doi:10.1002/jcc.20084 (2004).

76 Maier, J. A. et al. ff14SB: Improving the Accuracy of Protein Side Chain and Backbone Parameters from ff99SB. Journal of chemical theory and computation 11, 3696-3713, doi:10.1021/acs.jctc.5b00255 (2015).

77 Zgarbova, M. et al. Refinement of the Sugar-Phosphate Backbone Torsion Beta for AMBER Force Fields Improves the Description of Z- and B-DNA. Journal of chemical theory and computation 11, 5723-5736, doi:10.1021/acs.jctc.5b00716 (2015).

78 Jorgensen, W., Chandrasekhar, J. \& Madura, J. Comparison of simple potential functions for simulating liquid water. The Journal of chemical physics 79, doi: https://doi.org/10.1063/1.445869 (1983).

79 Essmann, U. et al. A smooth particle mesh Ewald method. The Journal of chemical physics 103, 8577-8593, doi:10.1063/1.470117 (1995).

80 Tamirat, M. Z., Koivu, M., Elenius, K. \& Johnson, M. S. Structural characterization of EGFR exon 19 deletion mutation using molecular dynamics simulation. PloS one 14, e0222814, doi:10.1371/journal.pone.0222814 (2019).

81 Roe, D. R. \& Cheatham, T. E., 3rd. PTRAJ and CPPTRAJ: Software for Processing and Analysis of Molecular Dynamics Trajectory Data. Journal of chemical theory and computation 9, 3084-3095, doi:10.1021/ct400341p (2013).

82 Humphrey, W., Dalke, A. \& Schulten, K. VMD: visual molecular dynamics. Journal of molecular graphics 14, 33-38, 27-38, doi:10.1016/02637855(96)00018-5 (1996).

83 Choi, H. et al. SAINT: probabilistic scoring of affinity purification-mass spectrometry data. Nature methods 8, 70-73, doi:10.1038/nmeth.1541 (2011).

84 Mellacheruvu, D. et al. The CRAPome: a contaminant repository for affinity purification-mass spectrometry data. Nature methods 10, 730-736, doi:10.1038/nmeth.2557 (2013).

85 Wang, J., Vasaikar, S., Shi, Z., Greer, M. \& Zhang, B. WebGestalt 2017: a more comprehensive, powerful, flexible and interactive gene set enrichment analysis toolkit. Nucleic acids research 45, W130-W137, doi:10.1093/nar/gkx356 (2017).

86 Meldal, B. H. et al. The complex portal--an encyclopaedia of macromolecular complexes. Nucleic acids research 43, D479-484, doi:10.1093/nar/gku975 (2015).

87 Giurgiu, M. et al. CORUM: the comprehensive resource of mammalian protein complexes-2019. Nucleic acids research 47, D559-D563, doi:10.1093/nar/gky973 (2019).

88 Buenrostro, J. D., Wu, B., Chang, H. Y. \& Greenleaf, W. J. ATAC-seq: A Method for Assaying Chromatin Accessibility Genome-Wide. Current 
bioRxiv preprint doi: https://doi.org/10.1101/732289; this version posted February 13,2020 . The copyright holder for this preprint (which was not certified by peer review) is the author/funder, who has granted bioRxiv a license to display the preprint in perpetuity. It is made available under aCC-BY-NC-ND 4.0 International license.

protocols in molecular biology 109, 2129 21-29,

1111 doi:10.1002/0471142727.mb2129s109 (2015).

1112 
bioRxiv preprint doi: https://doi.org/10.1101/732289; this version posted February 13,2020 . The copyright holder for this preprint (which was not certified by peer review) is the author/funder, who has granted bioRxiv a license to display the preprint in perpetuity. It is made

\section{Figure 1}

a

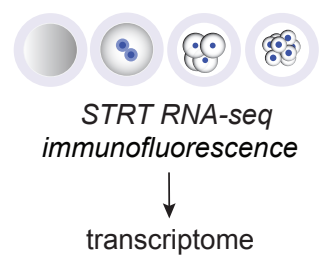

DUX4 protein localization

b

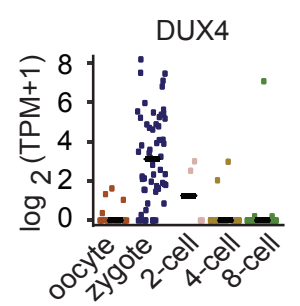

C

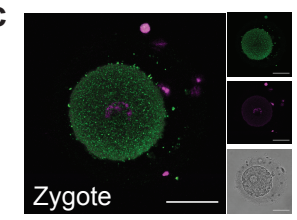

d

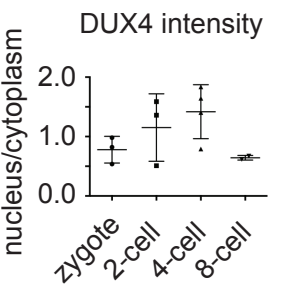

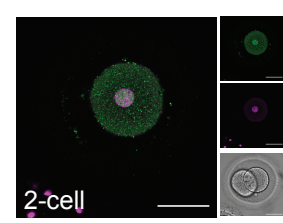
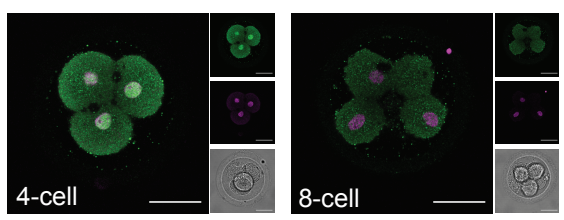

e SIRNACTRL / f SiRNA DUX4
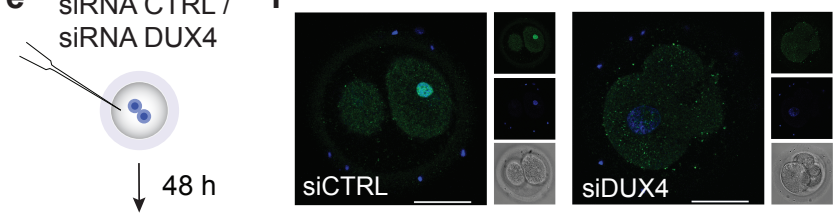

STRT RNA-seq

transcriptome g

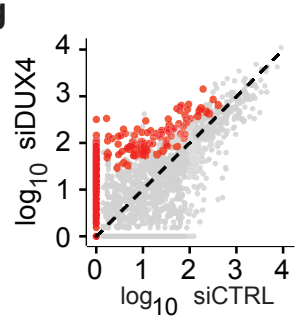

h

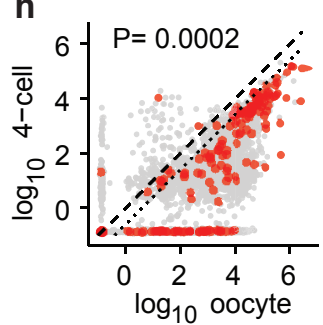

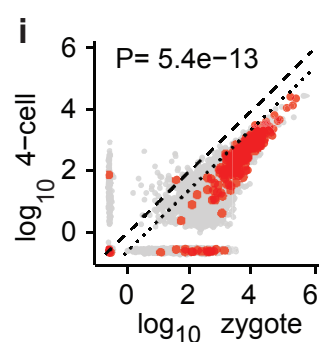

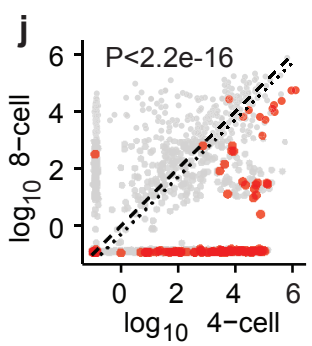

over

expressed

in siDUX4

NS

k

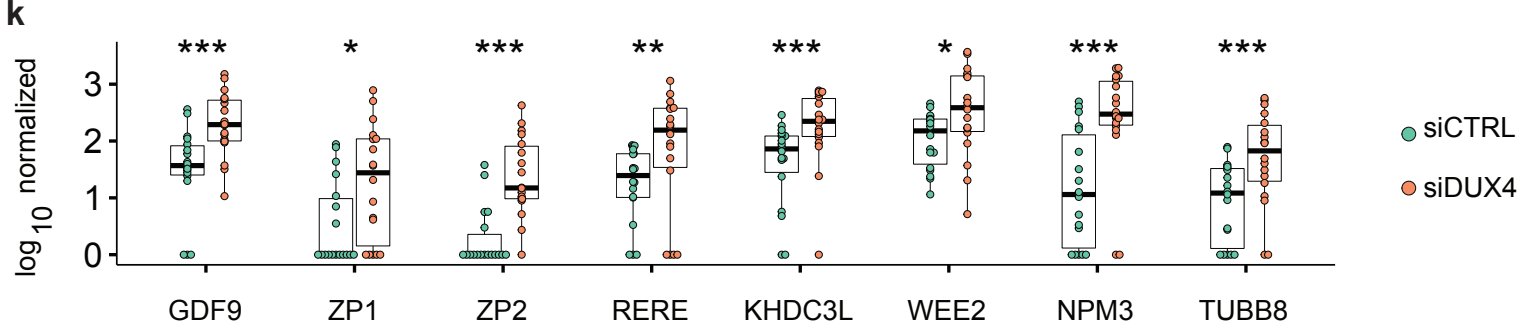

Figure 1. DUX4 knockdown leads to dysregulation of the maternal transcriptome in the human embryo.

(a) Schematic of the experimental design. (b) Log2 TPM (Transcripts Per Kilobase Million) of DUX4 mRNA reads in human MII oocytes ( $n=20$ ), zygotes $(n=59)$, 2-cell $(n=4), 4$-cell $(n=15)$, and 8-cell $(n=14)$ embryos8. (c) Representative confocal images of zygotes $(n=3), 2$-cell $(n=3) 4$-cell $(n=4)$, and 8-cell $(n=2)$ human embryos stained with monoclonal DUX4 antibody E5-5 (green). Nuclei counterstained with DAPI (magenta).

The larger left image of each panel shows the composite of the two small fluorescent images on the right for each stage. (d) Quantification of the DUX4 staining intensity in the nucleus normalized to the intensity in the cytoplasm. The samples as in 1c. Data are mean \pm SD. (e) siRNA experimental design. (f) Representative images of human embryos immunostained with DUX4 antibody (green) $24 \mathrm{~h}$ after microinjection with either control siRNA $(n=4)$ or DUX4 targeting siRNA $(n=5)$. Nuclei counterstained with DAPI (blue). Left side of each panel is a composite of individual corresponding z planes for DUX4 staining, nuclear staining, and the bright field channel (shown on the right side). Scale bar $50 \mu \mathrm{m}$. (g) Scatter plot of the expression levels of TFEs of siCTRL ( $n=18$ cells from two embryos) versus siDUX4 embryos ( $n=18$ cells from three embryos). Red dots represent significantly upregulated TFEs in siDUX4 embryos while grey dots represent TFEs with no significant change. (h-j) Using the oocyte to embryo transition data from Töhönen et al. 2015, we identified the TFEs present in our siCTRL versus siDUX4 data (g) and plotted them as follows h: oocyte to 4-cell, i: zygote to 4-cell, and j: 4-cell to 8-cell. The dotted line marks the cell division effect on cellular RNA content. The red dots are the upregulated TFEs identified in g. Note that they are downregulated in 4 cell embryos versus oocyte or zygote, and 8 cell versus 4 cell embryos, while in the DUX4 knock-down (siDUX4, g) they remain high, i.e. are not down regulated. P-values were calculated with Fisher's exact test for the frequency of the siDUX4-upregulated TFEs of the TFEs normally downregulated during respective stages. (k) Expression levels of selected oocyte-specific genes in SiCTRL and siDUX4 embryos. Wilcoxon test. Asterisks represent statistical significant changes.

${ }^{* * *} \mathrm{q}<0.001 ;{ }^{* *} \mathrm{q}<0.01 ;{ }^{*} \mathrm{q}<0.05$. Horizontal lines represent the median values in each group. 
bioRxiv preprint doi: https://doi.org/10.1101/732289; this version posted February 13, 2020. The copyright holder for this preprint (which was not certified by peer review) is the author/funder, who has granted bioRxiv a license to display the preprint in perpetuity. It is made Figure 2 available under aCC-BY-NC-ND 4.0 International license.

a TetOn DUX4 hESC

+ Dox

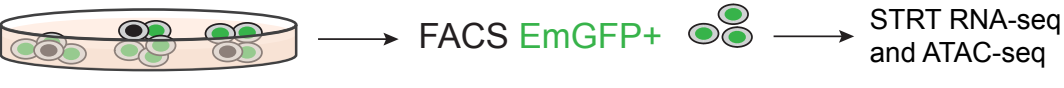

-Dox

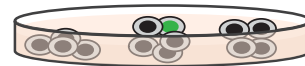

FACS EmGFP.

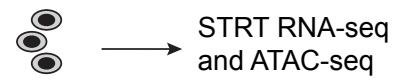

transcriptome and chromatin status

in EmGFP+ and

b

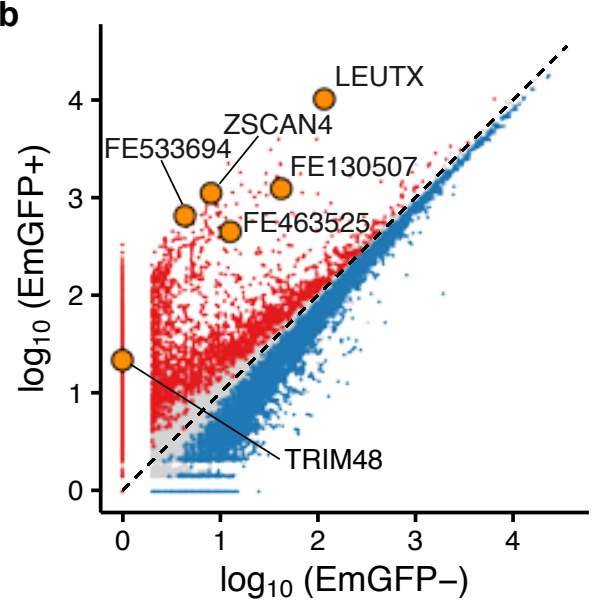

f

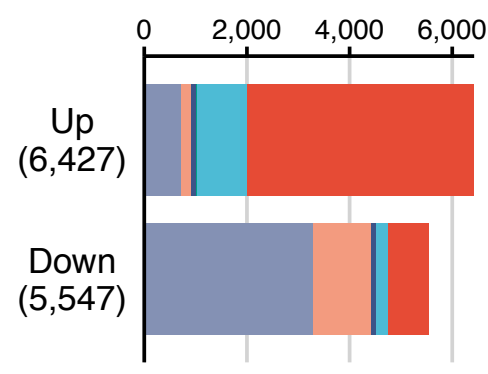

Upstream / 5'-UTR of coding

CDS / 3'-UTR of coding

Upstream $/ 1^{\text {st }}$ exon of noncoding

Other exons of noncoding

Intron

Unannotated
C

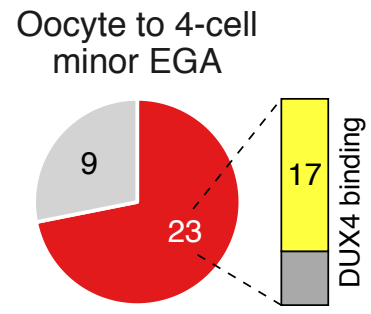

4- to 8-cell major EGA

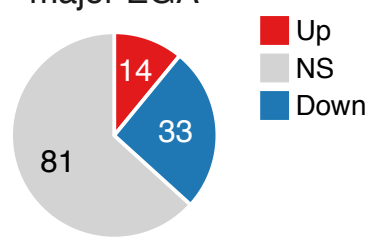

g

ATAC gained $(13,826)$

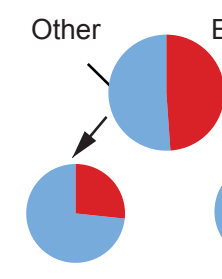

$26,7 \%$

overlap with DUX4 binding
ERVL-MaLR $48,9 \%$

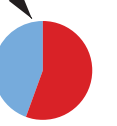

$55,8 \%$ **

overlap with DUX4 binding $\left({ }^{* *} \mathrm{P}<2.2 \mathrm{e}-16\right)$ d $\frac{\text { d }}{2}$

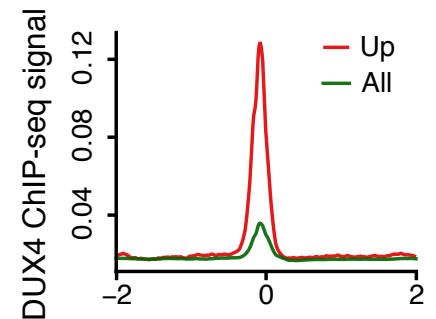

Distance from TFE peak $(\mathrm{kb})$

e Most enriched motif in Up

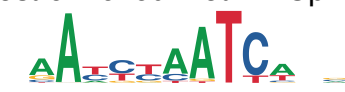
Known DUX4 motif 工А _.

ATAC

ATAC lost $(7,086)$ common $(32,019)$

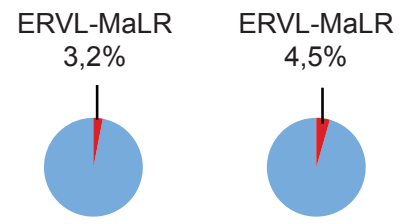

Figure 2. DUX4 causes upregulation of intergenic genome regions and minor embryo genome activation transcripts in human embryonic stem cells. (a) Schematic overview of the experimental design. (b) Overexpressed (red), downregulated (blue), and non-significantly changed (grey) TFEs after DUX4 induction (EmGFP $(+)$ cells. Three samples from two clones of each DUX4 TetOn hESC lines (H1 and H9) were FACS sorted and collected per indicated condition. (c) Proportion of the upregulated (Up), downregulated (Down), and non-significantly changed (NS) TFEs upon DUX4 induction as in (b) among the minor (oocyte to 4-cell embryo) and major (4- to 8-cell embryo) embryonic genome activation genes. One TFE out of the 129 major EGA genes annotated on an unassigned chromosome (ChrUn) and was excluded from the analysis. (d) DUX4 ChIP-seq intensity5 around the peaks of reads within the upregulated TFEs (red) and all the detected TFEs (grey). (e) De novo motif enrichment analysis of the DUX4-induced TFEs. Upper panel: the most significantly enriched motif $(P=1 \mathrm{e}-961)$ in upregulated (UP) genes using binomial statistical test. Lower panel: the best-matching known binding motif of DUX4 (DUX4 ChIP-seq of myoblasts: GSE7579179; matching score $=0.92$ ). (f) Positional information of the upregulated and downregulated TFEs after DUX4 induction. (g) Proportion of the gained, lost, and common ATAC-seq peaks overlapping ERVL-MaLR regions after DUX4 induction. The samples as in b. 
bioRxiv preprint doi: https://doi.org/10.1101/732289; this version posted February 13,2020 . The copyright holder for this preprint (which was not certified by peer review) is the author/funder, who has granted bioRxiv a license to display the preprint in perpetuity. It is made Figure 3

a TetOn DUX4 hESC

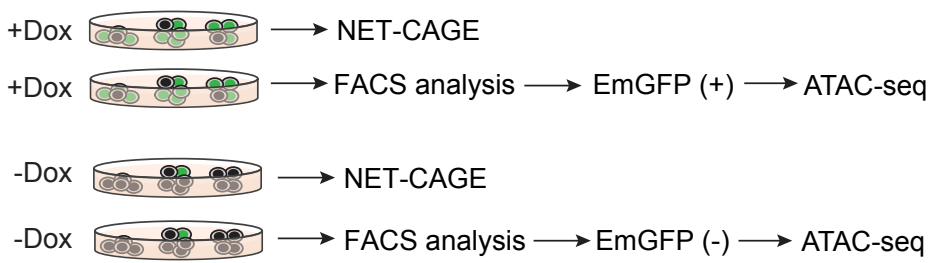

C

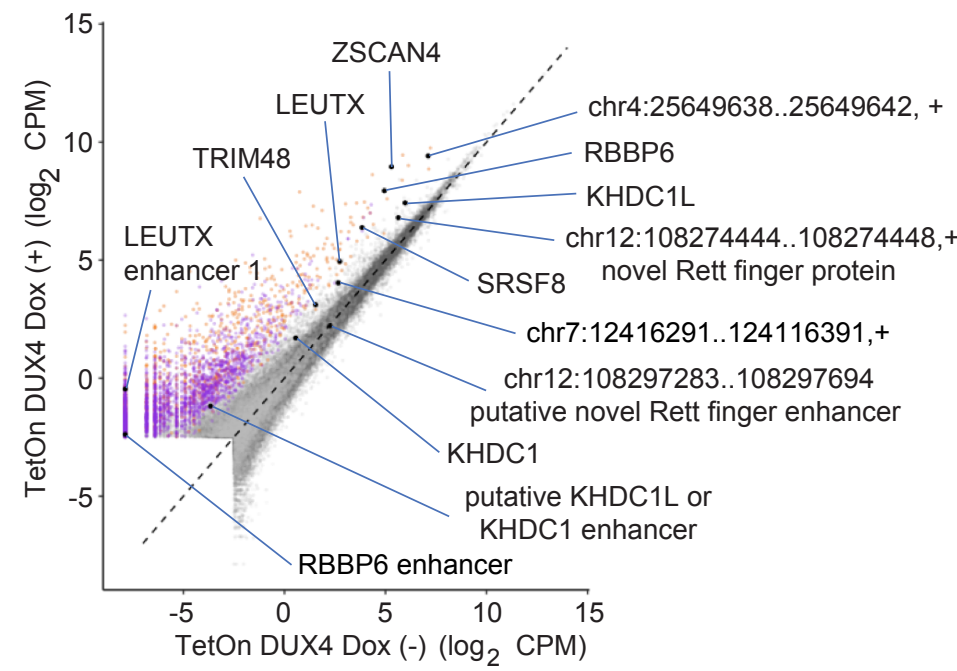

b TetOn DUX4 Dox (+) enhancers Dox (-) enhancers 4,013 / 10,502

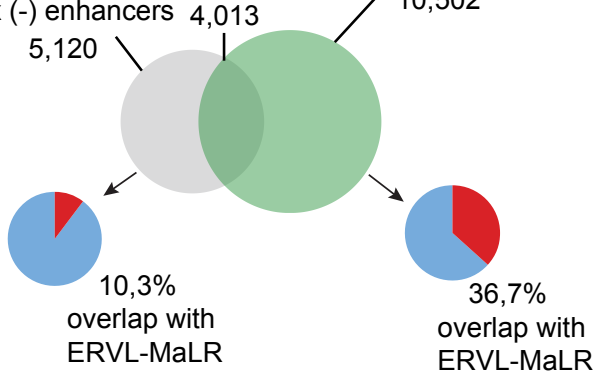

d

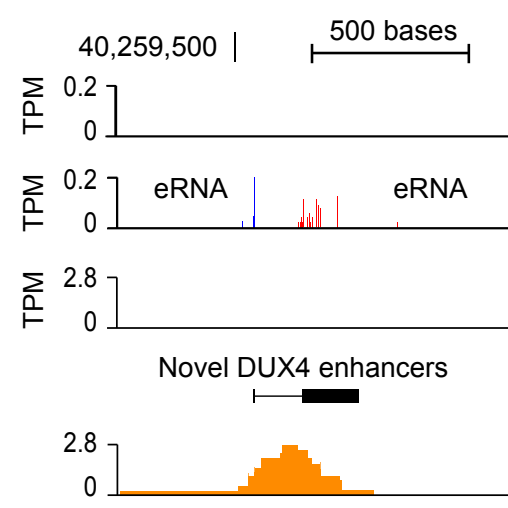

Dox (-) hESC NET-CAGE $\mathrm{fwd} / \mathrm{rev}$

Dox (+) hESC NET-CAGE $\mathrm{fwd} / \mathrm{rev}$

EmGFP (-) hESC ATAC-seq

EmGFP (+) hESC ATAC-seq
LEUTX promoter

$\begin{array}{ll}\text { - Enhancer } & \text { Enhancer NS } \\ \text { FDR }<0.01 & \mathrm{n}=12,994 \\ \mathrm{n}=2,166 & \\ \text { Promoter } & \text { • Promoter NS } \\ \text { FDR }<0.01 & \mathrm{n}=68,410 \\ \mathrm{n}=844 & \end{array}$

e

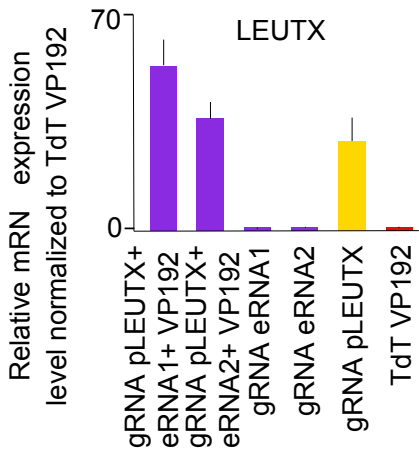

f

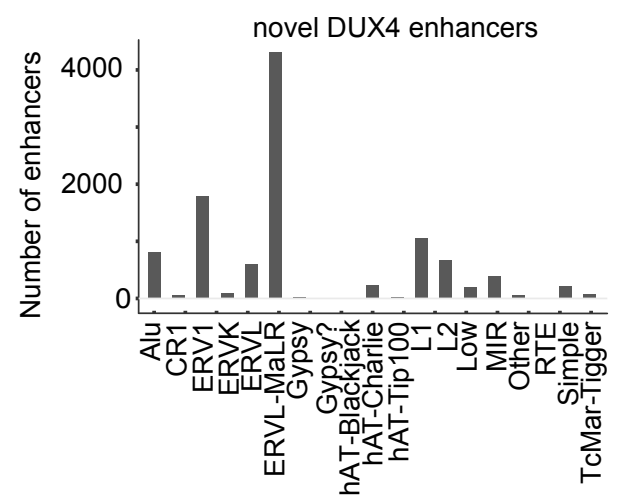

g

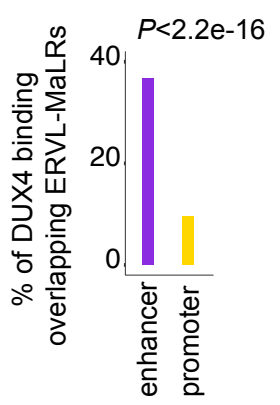

Figure 3. DUX4 activates newly identified enhancers of the minor embryo genome activation transcripts.

(a) Experimental design. (b) Venn diagrams of the novel transcribed enhancers identified in TetOn DUX4 Dox (-) ( $n=$ two biologically independent samples) and TetOn DUX4 Dox (+) ( $n=$ two biologically independent samples) cells. Pie charts show percentage of enhancers overlapping ERVL-MaLR repeat regions. (c) Comparison of TetOn DUX4 Dox (-) hESCs and TetOn DUX4 Dox (+) hESCs NET-CAGE data for promoters and enhancers. Lowly expressed promoters and enhancers with average expression < $-2.5 \log 2$ CPM in TetOn DUX4 Dox $(-)$ hESCs $(n=2)$ and TetOn DUX4 Dox (+) hESCs ( $=2)$ were filtered out. Yellow dots, differentially transcribed promoters (FDR $\leq 0.01$ ); purple dots, differentially transcribed enhancers (FDR $\leq 0.01$ ); dark grey dots, non-significant promoters; light grey dots, non-significant enhancers; black dots, promoters and enhancers for minor embryo genome activation genes. (d) NET-CAGE data shows bidirectional transcription for the putative LEUTX enhancer after DUX4 induction (the Dox (+) cells). ATAC-seq data illustrates open chromatin at the putative LEUTX enhancer region after DUX4 induction (the EmGFP $(+)$ cells). The LEUTX promoter14 was activated after DUX4 induction (the Dox (+) cells, correlating with open chromatin region (ATAC-seq peaks in the EmGFP (+) cells). (e) Relative expression level of LEUTX in HEK293 cells transfected with the indicated guide RNAs and plasmids. gRNA, guide RNA; eRNA, enhancer RNA; VP192; dCas9 transactivator plasmid; pLEUTX, LEUTX promoter. (f) Novel DUX4 enhancers overlapping with retroelement families. The retroelement families overlapping with at least ten DUX4 enhancers are shown. (g) Proportion of the DUX4 binding sites overlapping ERVL-MaLRs at enhancer or promoter regions. 
bioRxiv preprint doi: https://doi.org/10.1101/732289; this version posted February 13,2020 . The copyright holder for this preprint (which was not certified by peer review) is the author/funder, who has granted bioRxiv a license to display the preprint in perpetuity. It is made available under aCC-BY-NC-ND 4.0 International license.

\section{Figure 4}

a

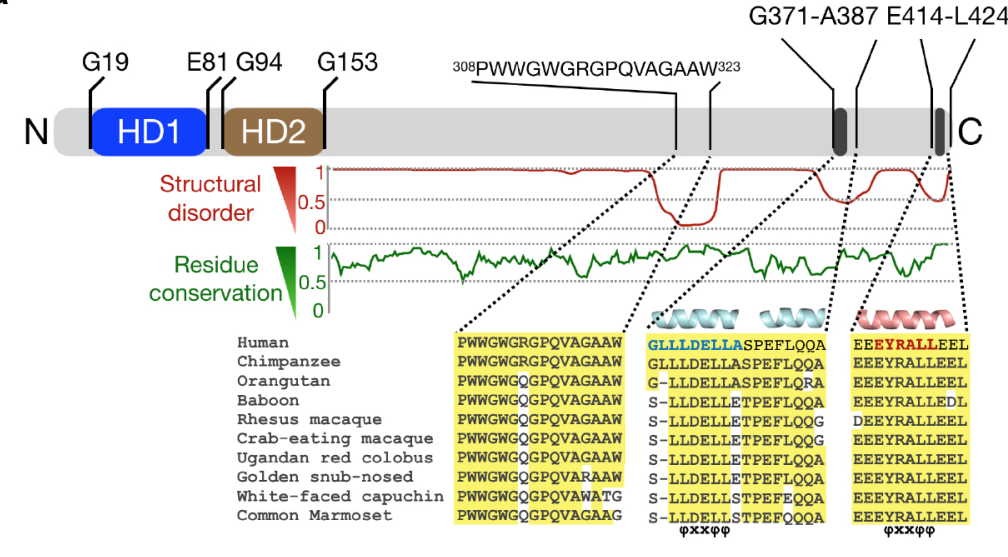

b

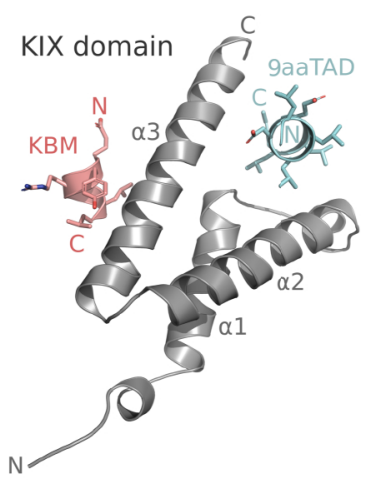

C

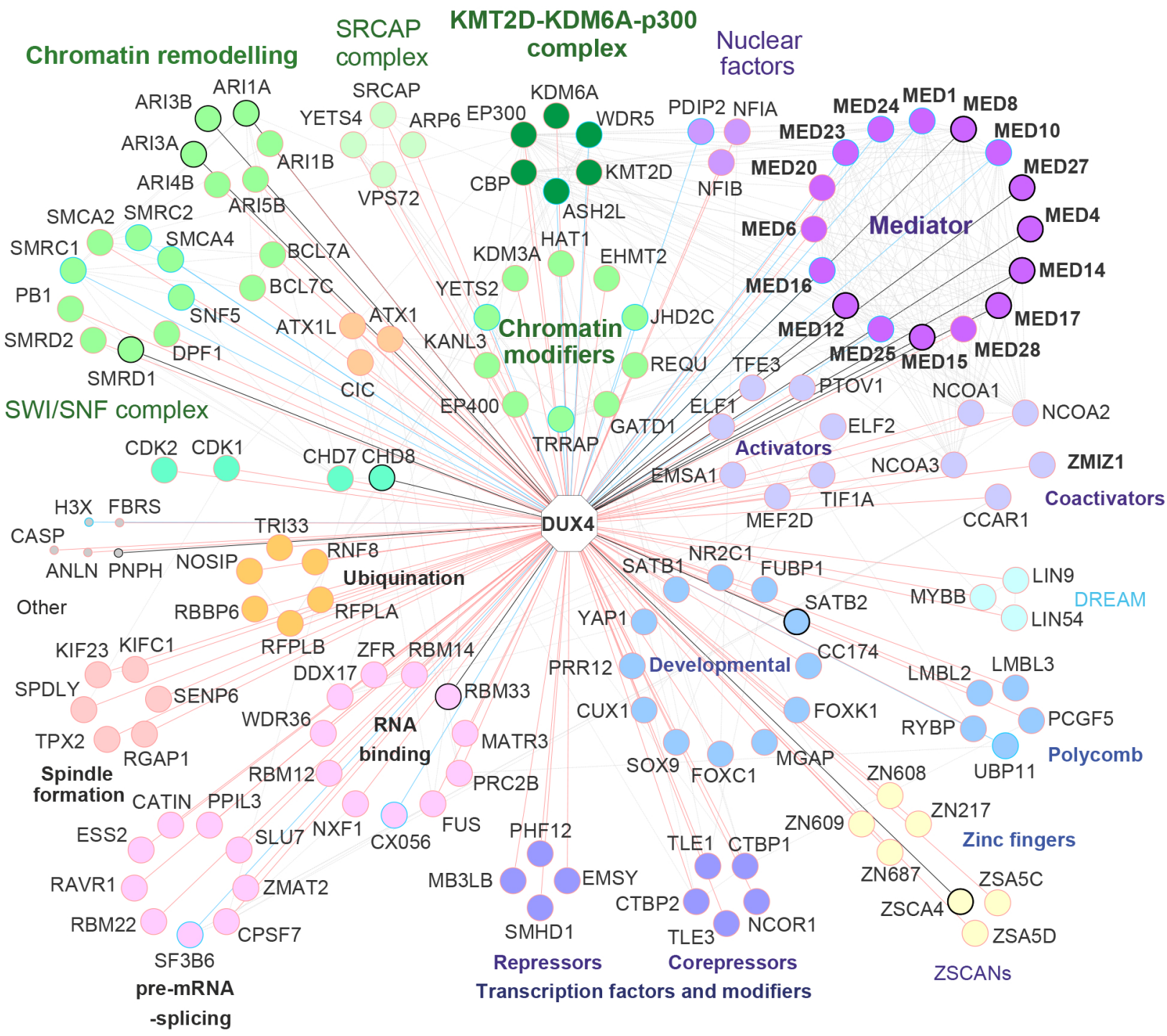

Figure 4. C-terminus of human DUX4 interacts with the transcriptional Mediator complex and chromatin regulators.

(a) Domain structure of full-length DUX4: N-terminal homeodomains HD1 and HD2, and C-terminal region. Conservation of residues in primates versus human sequences (green curve) C-terminal to residue G153 and sequence alignment of three conserved regions with a disorder value lower than 0.5 (red curve). Residue numbering from UniProt ID Q9UBX2. Two helical regions are predicted within the C-terminal region, the first one (cyan helices) and the second one (salmon helix) both containing the amphipathic "ФXXФФ" motif ( $\Phi$, bulky hydrophobic amino acid; $X$, any amino acid) found in several transcription factors reported to interact with KIX80-82. The position of the 9aaTAD (blue letters) and KBM (KIX binding motif; red letters) sequences are indicated by black bars. (b) Modelled interactions of the human KIX domain (PDB: 2LXT) with DUX4 9aaTAD (cyan) and KBM (salmon). (c) DUX4 protein-protein interactome. BiolD -interactions shown with red lines and AP-MS -interactions with blue lines. If protein appeared in both data sets it is outlined in bold black. Known prey-prey interactions shown in grey (iREF). 
bioRxiv preprint doi: https://doi.org/10.1101/732289; this version posted February 13, 2020. The copyright holder for this preprint (which was not certified by peer review) is the author/funder, who has granted bioRxiv a license to display the preprint in perpetuity. It is made Extended Data Fig. 1

a

b

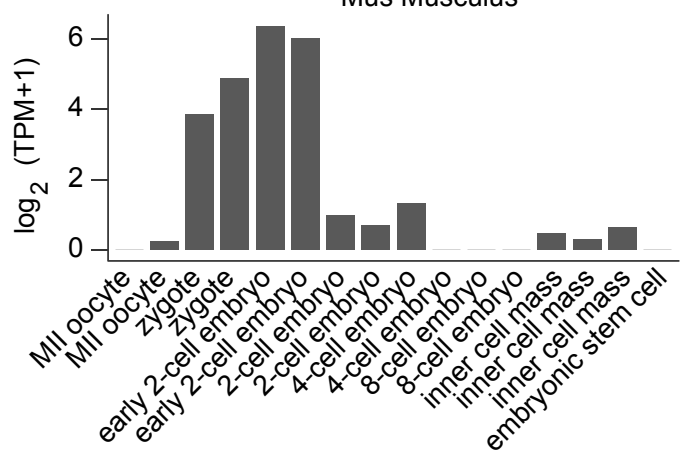
available under aCC-BY-NC-ND 4.0 International license.
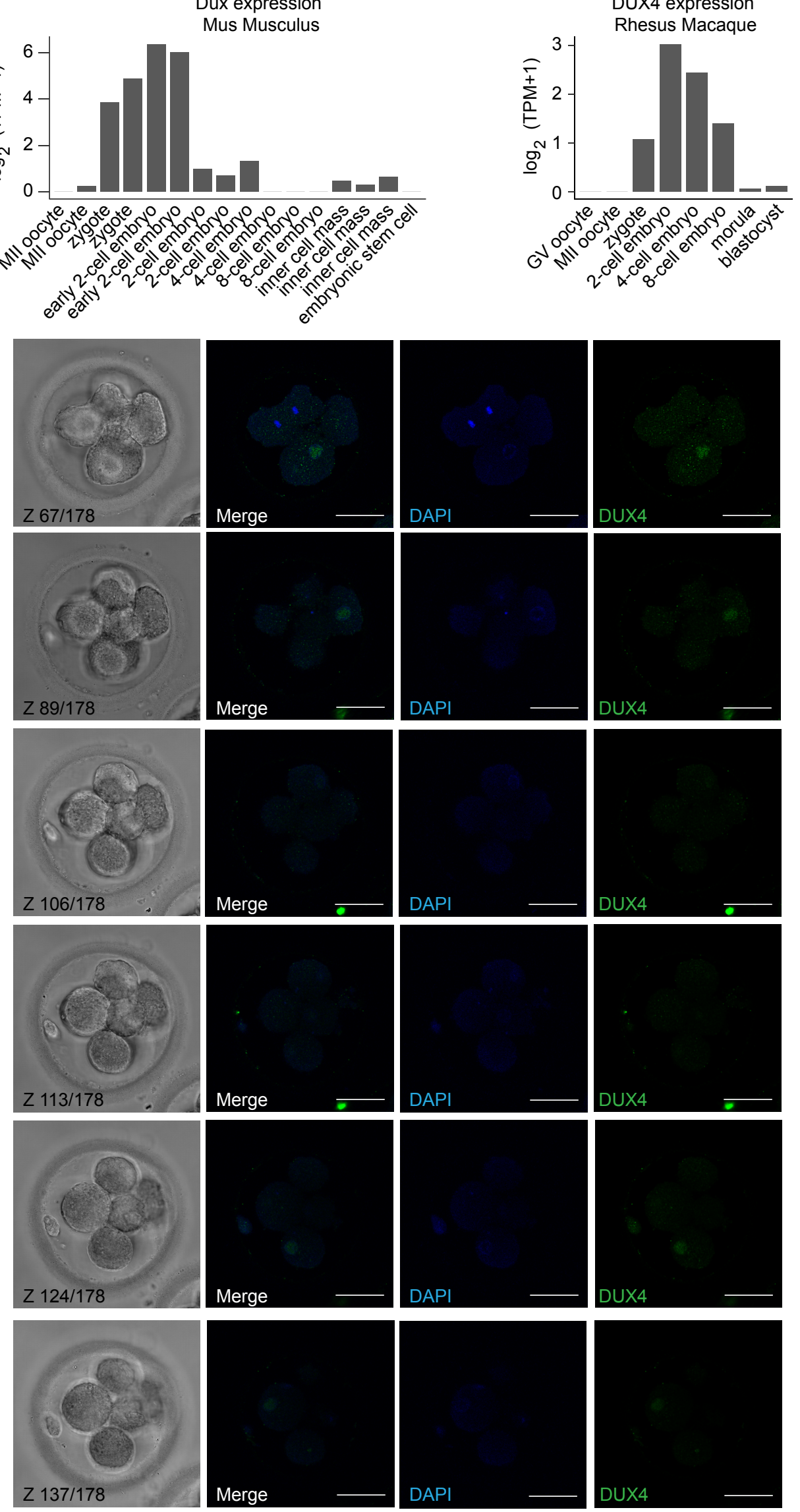
bioRxiv preprint doi: https://doi.org/10.1101/732289; this version posted February 13,2020 . The copyright holder for this preprint (which was not certified by peer review) is the author/funder, who has granted bioRxiv a license to display the preprint in perpetuity. It is made

Extended Data Fig. 2

a

CL:0000021_female germ

CL:0000023_oocyte

CL:0000039_germ line cell

CL:0000586_germ cell UBERON:0001828_gingiva

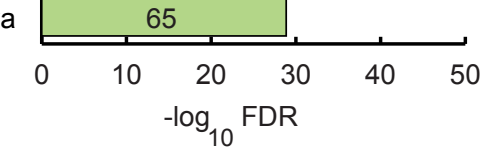

C

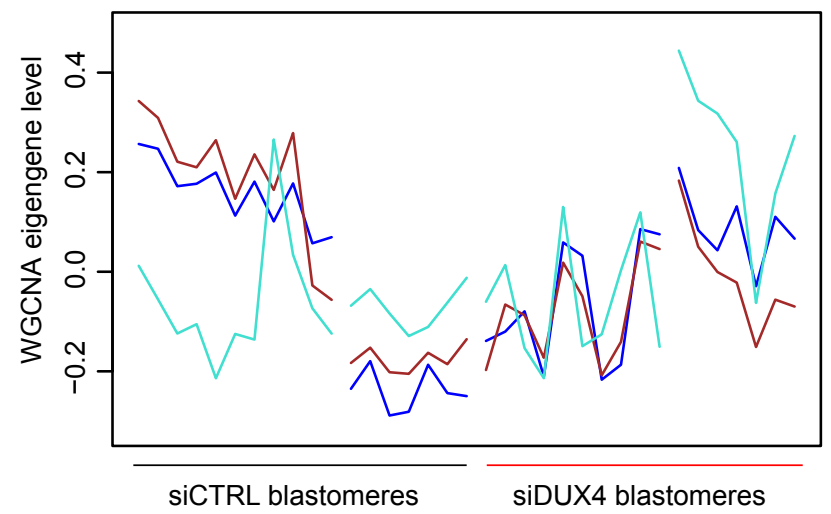

Blastomeres in embryos b Cluster dengrogram

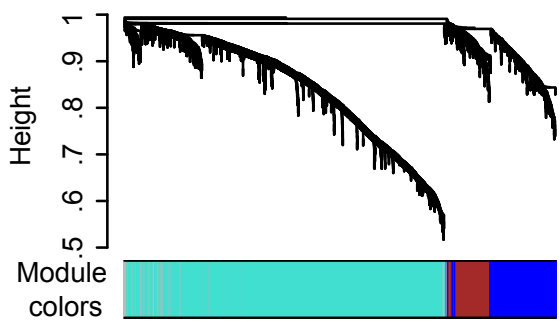

Extended Data Figure 2. DUX4 knockdown leads to insufficient genome activation.

(a) Gene expression enrichment analysis for the genes that were retained in the siDUX4 embryos using TopAnat. (b) Hierarchical clustering and module assignment of the 3,196 variable TFEs between the SiCTRL and siDUX4 embryos. A maternal (turquoise) and two embryo genome activation gene modules (blue and brown) were assigned. (c) Representative expression levels of each module as indicated in (b) by the siCTRL and siDUX4 blastomeres. (d) TFEs and annotations by modules and locations. 
bioRxiv preprint doi: https://doi.org/10.1101/732289; this version posted February 13,2020 . The copyright holder for this preprint (which was not certified by peer review) is the author/funder, who has granted bioRxiv a license to display the preprint in perpetuity. It is made

\section{Extended Data Fig. 3}

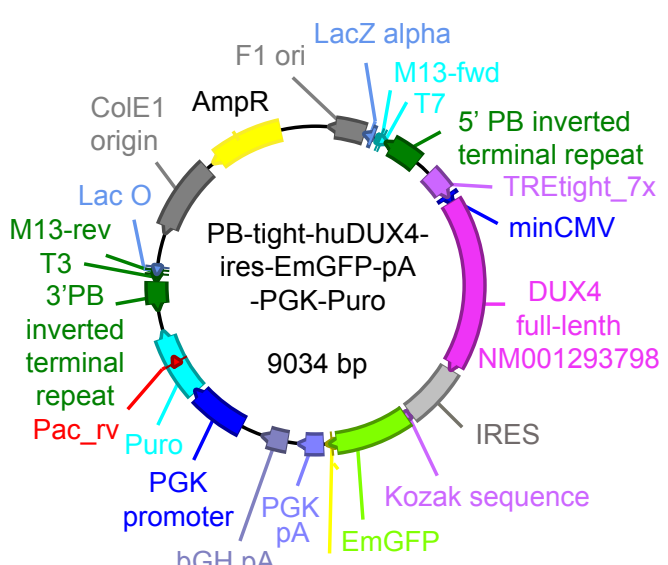

+ pB-CAG-rtTA

+ pBase (transposase)
C

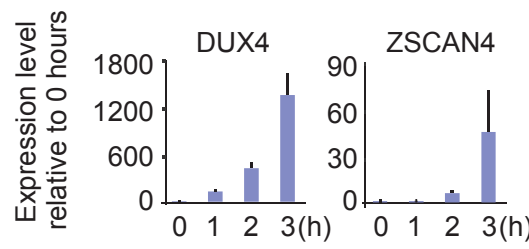

Enrichment analysis with ChIP-seq datasets

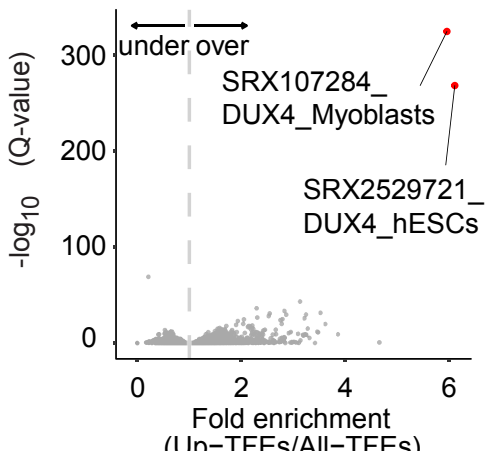

b
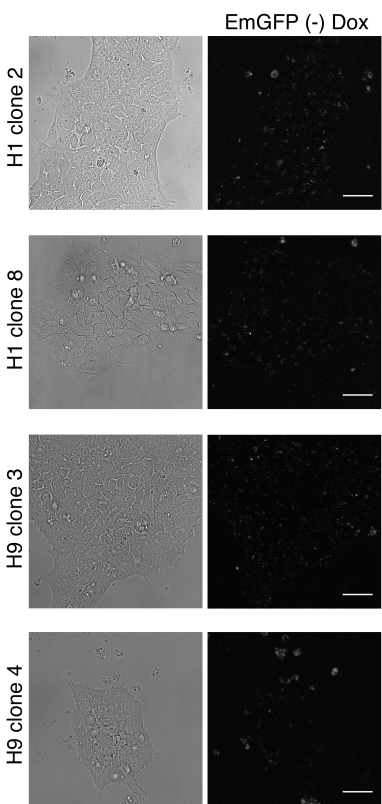

(-) Dox

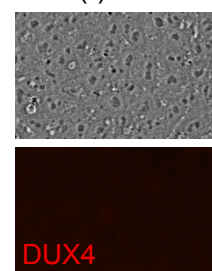

$\operatorname{EmGFP}(+)$ Dox
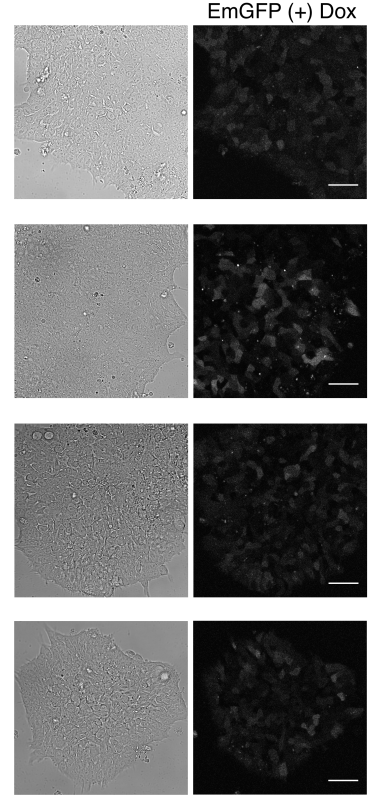

(+) Dox
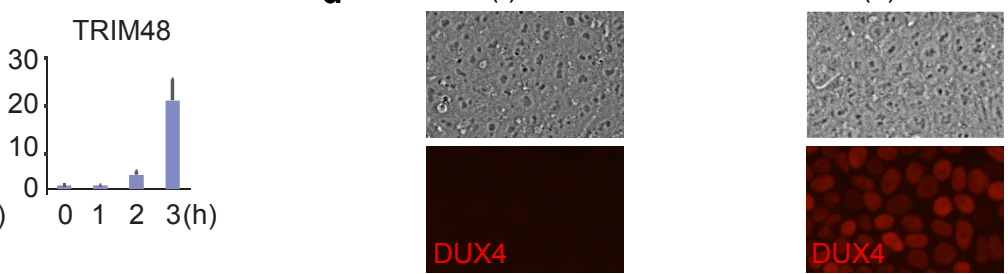

f

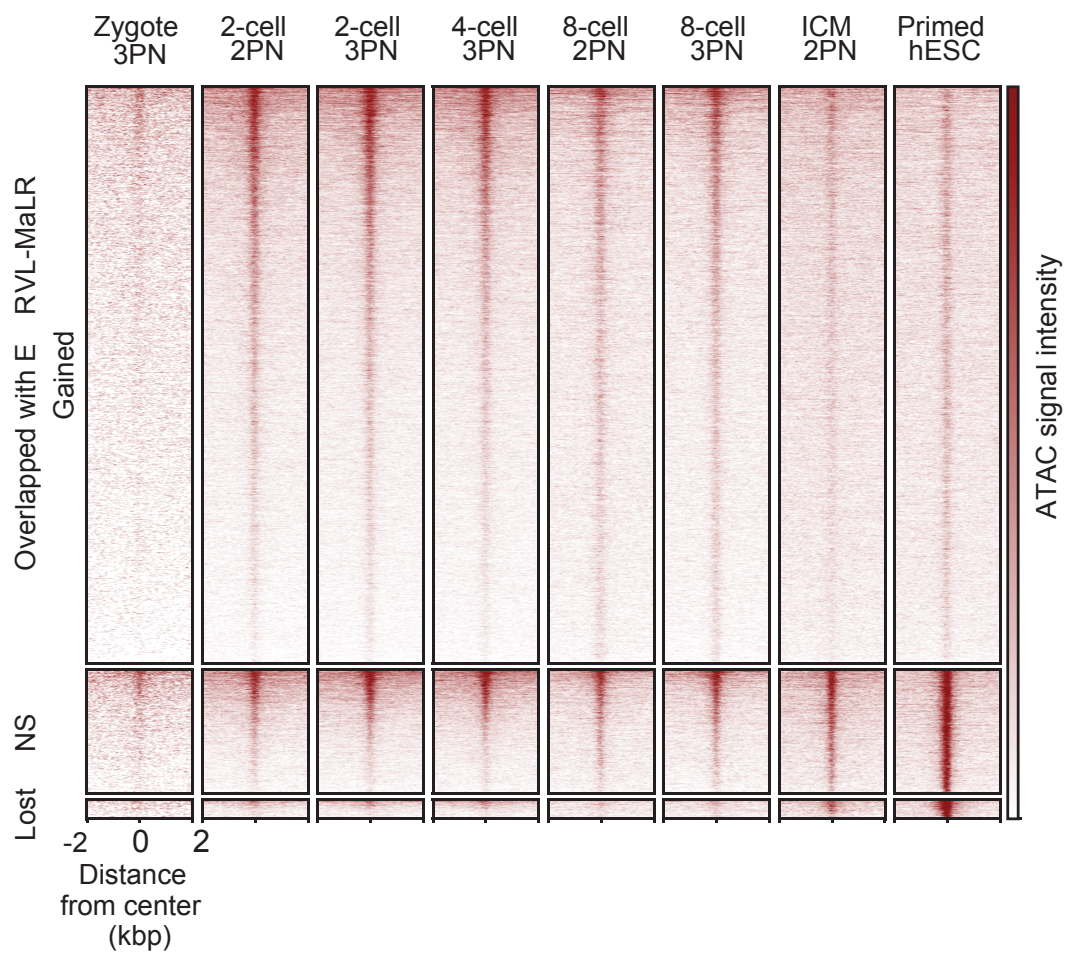

Extended Data Figure 3. Induction of DUX4 in the DUX4 TetOn hESCs leads to expression of intergenic genome.

(a) The DUX4-ires-EmGFP piggyBac vector used to establish the doxicycline inducible DUX4 TetOn hESCs in $\mathrm{H} 1$ (clones 2 and 8 ) and $\mathrm{H} 9$ (clones 3 and 4 ). (b) DUX4 TetOn hESC clones (as above) $+/-1 \mu \mathrm{g} / \mathrm{ml}$ doxicycline for $3 \mathrm{~h}$ and live imaged for EmGFP. (c) mRNA expression kinetics of DUX4, ZSCAN4, and TRIM48 after $1 \mathrm{~h}, 2 \mathrm{~h}$, and $3 \mathrm{~h}$ doxicycline induction measured using qRT-PCR. The data for the DUX4 TetOn H1 clone 2 is shown. Similar expression patterns were also found for the $\mathrm{H} 1$ clone 8 , and $\mathrm{H} 9$ clones 3 and 4 . (d) DUX4 TetOn hESCs treated with $1 \mu \mathrm{g} / \mathrm{ml}$ doxicycline for $4 \mathrm{~h}$, fixed, and immunostained for DUX4. Representative images for nuclear DUX4 staining are shown for the $\mathrm{H} 1 \mathrm{clone} 2$. Similar staining pattern were seen for the $\mathrm{H} 1$ clone 8 and $\mathrm{H} 9$ clones 3 and 4 . Scale bar $50 \mu \mathrm{m}$. (e) Enrichment analysis of the DUX4-induced TFEs with 816 publicly available ChIP-seq datasets. A total of 7,216 ChIP-seq data for transcription factors are shown. ChIP-seq data for DUX4 are shown in red. Dots on the left side of the dashed line are underrepresented, whereas dots on the right side are overrepresented. (f) ATAC-seq intensity of human early embryo around the gained, non-significant (NS), and lost ATAC-seq peaks after DUX4 induction which overlap with ERVL-MaLR elements. 
bioRxiv preprint doi: https://doi.org/10.1101/732289; this version posted February 13, 2020. The copyright holder for this preprint (which was not certified by peer review) is the author/funder, who has granted bioRxiv a license to display the preprint in perpetuity. It is made available under aCC-BY-NC-ND 4.0 International license.

\section{Extended Data Fig.4}

a KHDC1 pseudo gene 1

ENA accession LR694084, LR694085

500 bases

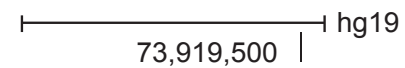

$73,920,000$

TFE; Töhönen et al. $2015^{8}$

TFE TetOn-DUX4

Transcripts by assemle on TetON-DUX4

DUX4 ChIP*

cDNA clone

Human mRNA Genbank

Human ESTs

AK054891

DB296853

FE463525

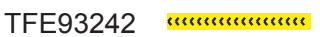

b RING-finger type E3 ubiquitin ligase ENA accession LR694082, LR694083

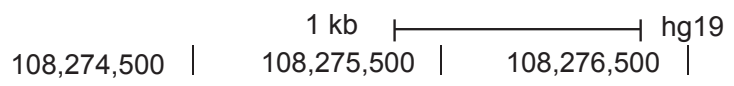

TFE; Töhönen et al. $2015^{8}$

FE130507

TFE TetOn-DUX4

Transcripts assemble TetON-DUX4

DUX4 ChIP*

cDNA clone

TFE25640 'm”'

Human mRNA Genbank

Human ESTs

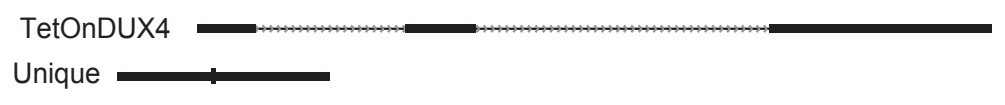

RET11.1

KJ893228

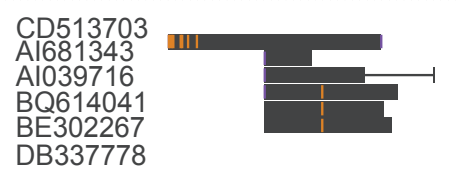

C RING-finger domain protein

ENA accession:

LR694086, LR694087 (Ring10.2)

LR694088, LR694089 (Ring4.2)

TFE; Töhönen et al. $2015^{8}$

TFE TetOn-DUX4

Transcripts assemble TetON-DUX4

DUX4 ChIP*

cDNA clones

Human mRNA Genbank

Human ESTs

K5.2

$108,275,500$

Extended Data Figure 4. Previously unannotated putative DUX4 target genes cloned from cDNA of a human 4-cell embryo.

(a) Predicted KHDC1 pseudo gene 1 (clone K5.2), at chromosome $6(73,918,461-824$ 73,920,115) was expressed by human 4-cell embryos (FE463525) and upregulated in TetOn DUX4 hESCs (TFE93242). TFEs overlapped with DUX4 binding sites (DUX4 ChIP). cDNA clone K5.2 (thick orange regions indicate exons and grey thin regions indicate introns) corresponds to the KHDC1 pseudogene 1 transcript assembly in TetOn DUX4 cells. Transcript assemblies (mRNA Genbank and human ESTs), including unspliced, are shown. (b) Putative RING-finger type E3 ubiquitin ligase at chromosome 2 $(108,273,771-831$ 108,277,850) was expressed by human 4-cell embryos (FE130507) and it was upregulated in TetOn-DUX4 hESCs (TFE25640). The DUX4 ChIP-seq peak overlapped with the TFEs. RET11.1 was cloned from human 4-cell embryo (clone RET11.1). Thick blue regions indicate exons and thin grey regions indicate introns. Transcript assemblies (mRNA Genbank and human ESTs), including unspliced, are shown. (c) Putative RING-finger domain protein at chromosome 8 (210,701-215,100) was expressed by human 4-cell embryos (TFE533694) and induced in TetOn-DUX4 hESCs

(TFE102707). ChIP-seq overlapped with the TFEs. Two cDNA clones, Ring 4.2 and Ring 10.22, were expressed in the human 4-cell embyos. Thick blue regions indicate exons and grey thin regions indicate introns. Transcript assemblies (mRNA Genbank and human ESTs), including unspliced, are shown. 
bioRxiv preprint doi: https://doi.org/10.1101/732289; this version posted February 13,2020 . The copyright holder for this preprint (which was not certified by peer review) is the author/funder, who has granted bioRxiv a license to display the preprint in perpetuity. It is made Extended Data Fig. 5
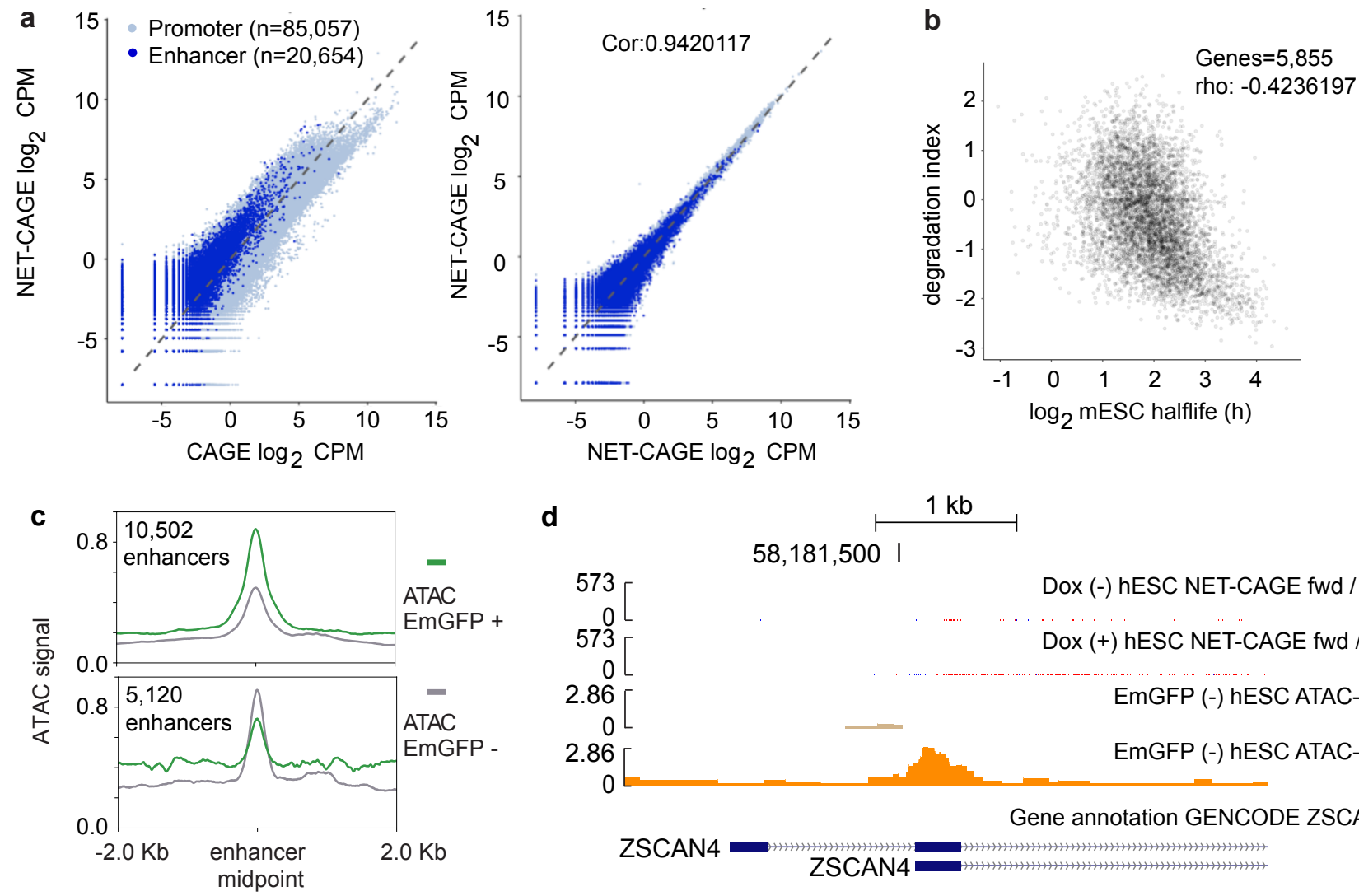

d
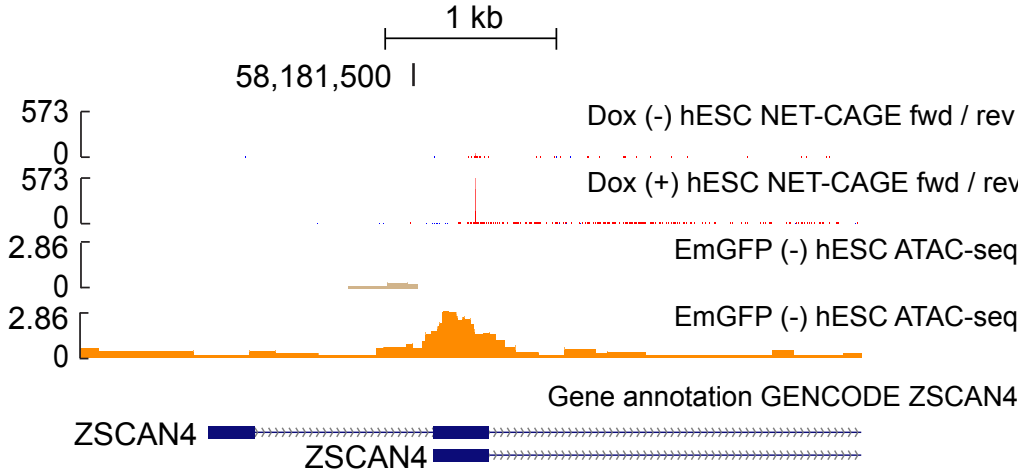

e scale

chrom 19

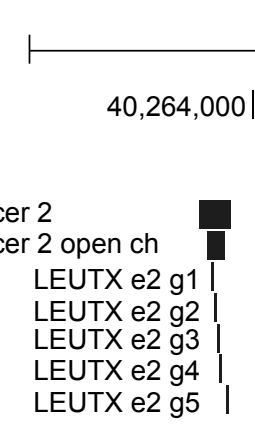

$5 \mathrm{~kb}$

$40,260,000$

enhancer 2

enhancer 1

enhancer 1 open ch

LEUTX e1 g1

LEUTX e1 g2

LEUTX e1 g3

LEUTX e1 $\mathrm{g} 4$

LEUTX e1 g5

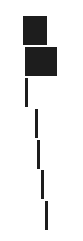

enhancer 2 open ch

LEUTX e2 g3

LEUTX e2 g5 |

Dox (-) hESC NET-CAGE fwd / rev

EmGFP (-) hESC ATAC-seq

EmGFP (-) hESC ATAC-seq

f

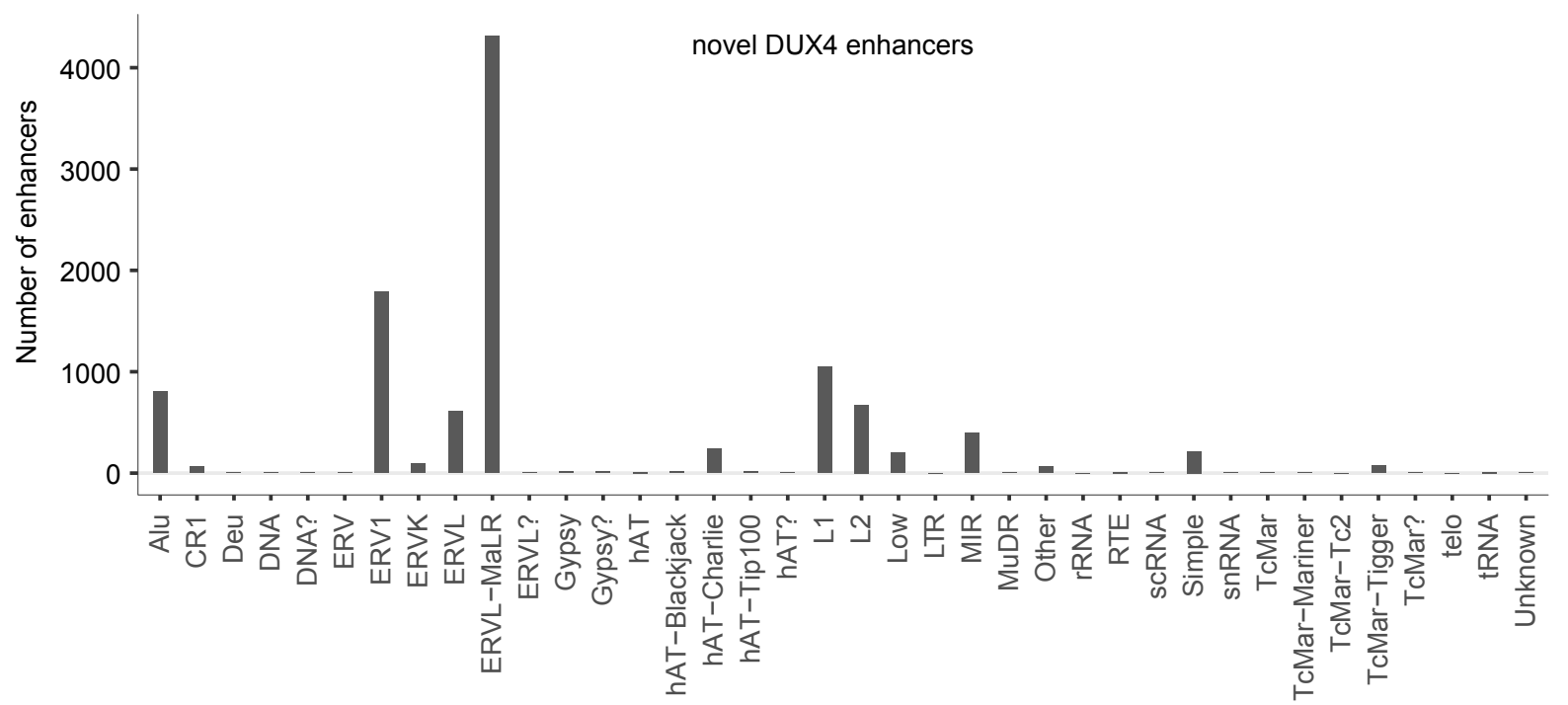

Extended Data Figure 5. DUX4 activates novel enhancers.

(a) Comparison of CAGE and NET-CAGE data in DUX4 TetOn hESCs. Unstable nascent RNA transcripts, like enhancer RNAs, were detected with high sensitivity using NET-CAGE in comparison to CAGE. (b) Scatter plot of log2 (half-lives) and degradation indices calculated as log2 (NET-CAGE/CAGE) ratios in TetOn DUX4 hESC control sample and mouse embryonic stem cells. Each dot represents a gene. (c) Metagene plots showing ATAC-seq signal for the novel DUX4 enhancers and control only enhancers in the EmGFP (+) and EmGFP (-) cells. (d) UCSC browser view of the ZSCAN4 promoter identified using NET-CAGE. NET-CAGE signal shown in the DUX4 TetOn hESCs with and without Doxicycline treatment. Chromatin status (ATAC-seq signal) shown in the EmGFP (-) and EmGFP (+) cells. (e) Positions of the CRISPR guide RNAs used for LEUTX activation. e1, enhancer 1; e2, enhancer 2; g, guide; EP, embryo promoter; open ch, open chromatin. (f) Novel DUX4 enhancers overlapping with retroelement families. All families overlapping with at least one enhancer are shown. 
bioRxiv preprint doi: https://doi.org/10.1101/732289; this version posted February 13, 2020. The copyright holder for this preprint (which was not certified by peer review) is the author/funder, who has granted bioRxiv a license to display the preprint in perpetuity. It is made available under aCC-BY-NC-ND 4.0 International license.

\section{Extended Data Figure 6}

a
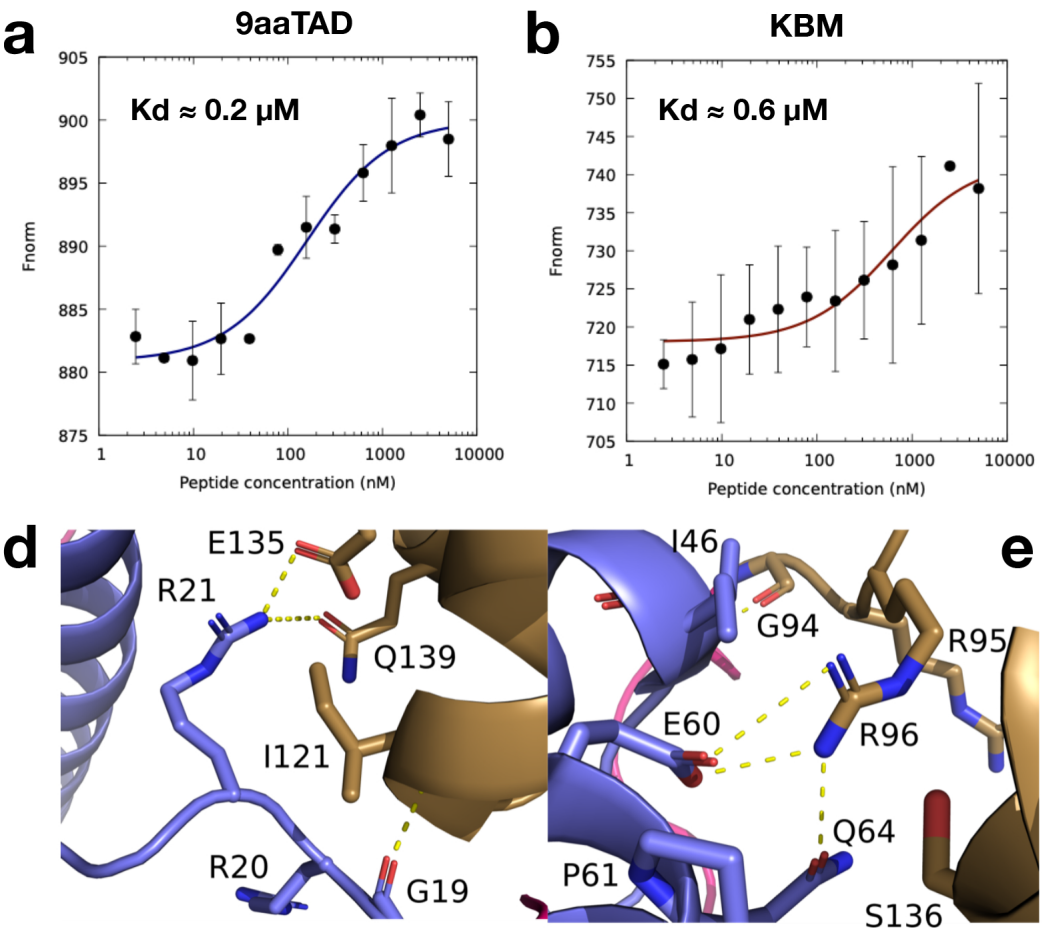

e

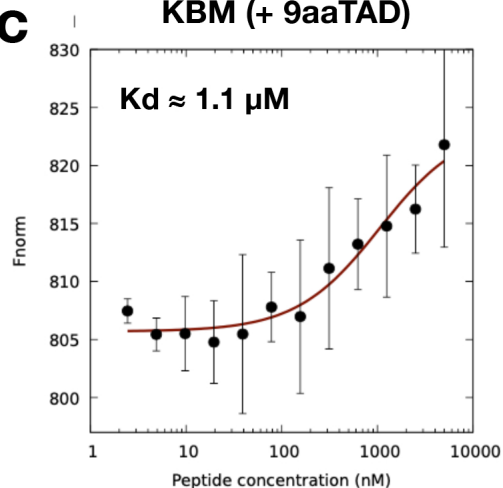

HD numbering $1 \quad 3 \quad 28424346$

Human DUX4 numbering 192146606164
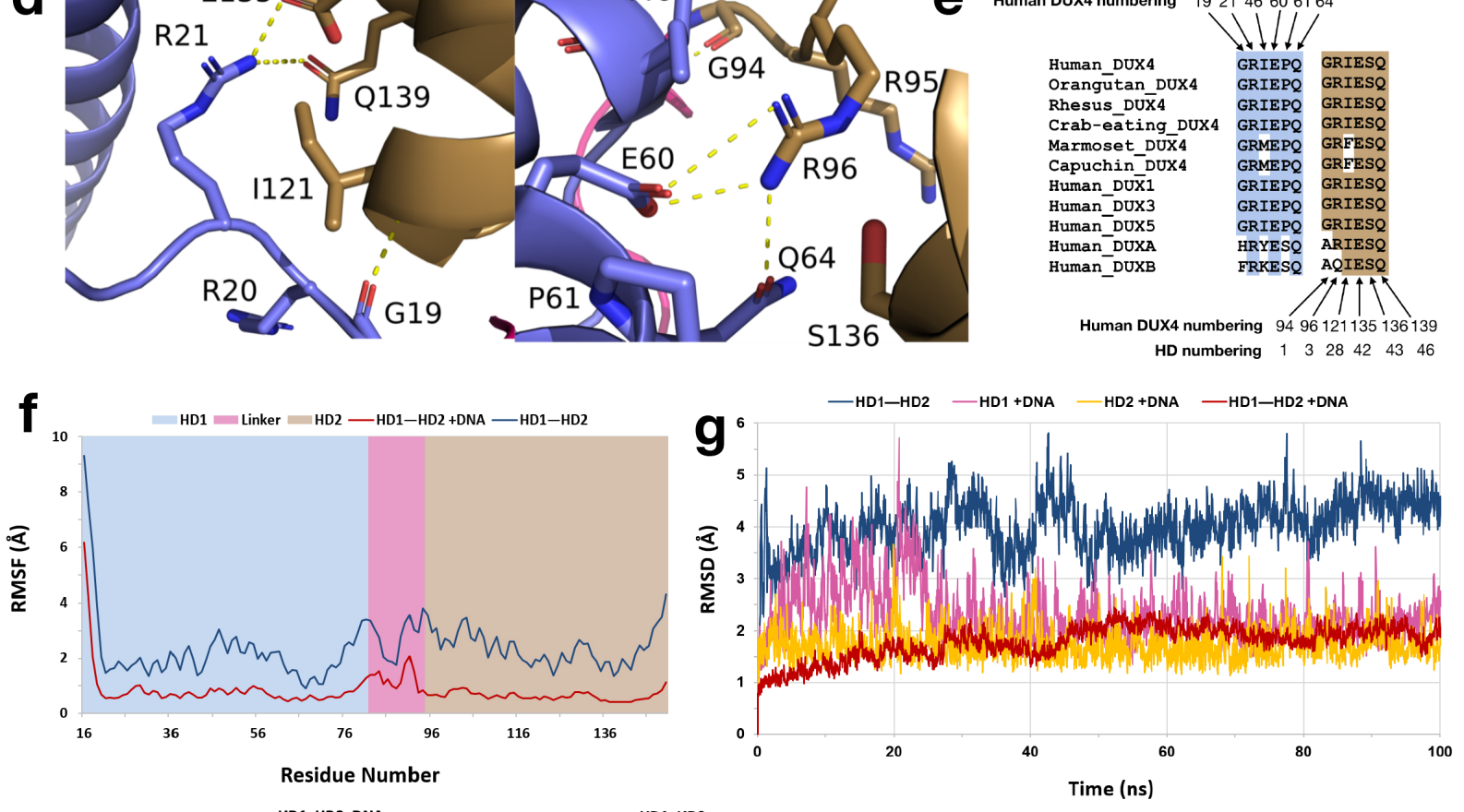

h
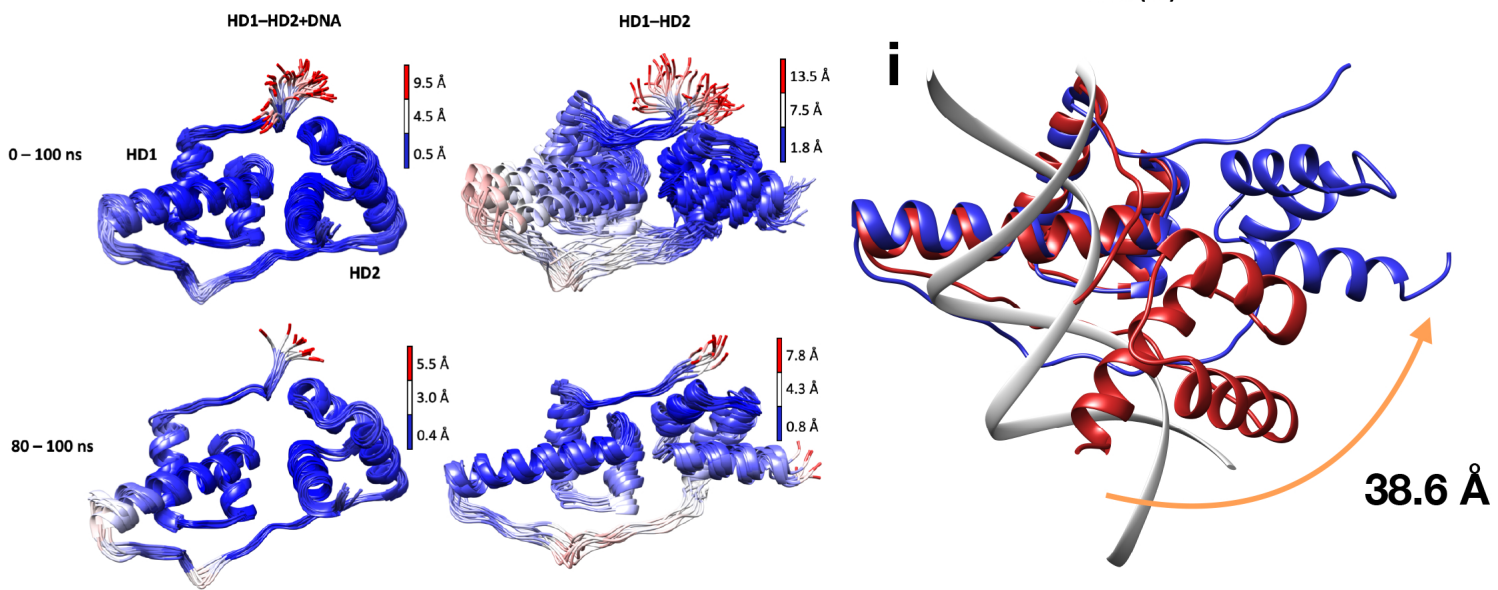

Extended Data Figure 6. Interactions of DUX4. Microscale thermophoresis binding analysis of peptides to human KIX domain.

(a) 9aaTAD peptide (C370-Q386) (b) KBM peptide (E414-E423) (c) KBM binding to KIX with saturating 9aaTAD. (d) Inter-HD interactions stabilizing DUX4 HD1 and HD2 in absence of bound DNA (e) Sequence comparison of HD1-HD2 interacting residues seen in human DUX4 with other primates and other human double HD transcription factors. (f) RMSF (Ca atoms) of X-ray structure of DUX4 with (red curve) and without (blue curve) bound DNA during a 100 ns MD simulation. HD1 (blue), linker (magenta) and HD2 (gold). (g) RMSD (backbone atoms) with reference to starting conformation) of X-ray structure of DUX4 HD1-HD2 with and without bound DNA, and separately for HD1 and for HD2 with bound DNA, during 100 ns MD simulations. (h) Superposed conformations of DUX4 with (left) and without (right) DNA, sampled during $100 \mathrm{~ns}$ (top) and final $20 \mathrm{~ns}$ (bottom) of the simulation. Chain traces are colored based on the Ca-atom RMSD relative to the median structure at $50 \mathrm{~ns}$ or 90 ns. DNA-bound DUX4 shows higher stability than DNA-free DUX4; both exhibit larger fluctuations at the unconstrained N-termini and linker loops. A more stable conformation of DNA-free DUX4 exposing residues of the recognition helices was attained during the last $20 \mathrm{~ns}$. (i) Final pose, DNA-free DUX4 (blue), after $100 \mathrm{~ns}$ simulation with HD1 superposed on HD1 of DNA-bound DUX4 X-ray structure (red and grey), revealing the degree of "opening" seen in the simulation; e.g. the Ca-atom of $\mathrm{R} 146$ of the third helix of HD2 differs in relative position by $38.6 \AA$. 
bioRxiv preprint doi: https://doi.org/10.1101/732289; this version posted February 13,2020 . The copyright holder for this preprint (which was not certified by peer review) is the author/funder, who has granted bioRxiv a license to display the preprint in perpetuity. It is made available under aCC-BY-NC-ND 4.0 International license.

Extended Data Table 7. Supplementary table showing oligos that were used to (a) target LEUTX in a CRISPRa assay (b) measure expression levels of indicated genes in quantitative real-time PCR .

(a) Guide oligos for LEUTX promoter and enhancer. The guide RNA sequence is underlined. p:promoter; g:guide; enh:enhancer.

\begin{tabular}{|l|l|}
\hline LEUTX_p_g2 & GTGGAAAGGACGAAACACCGgcgtggtattagggtaggacGTTTTAGAGCTAGAAATAG \\
\hline LEUTX_p_g3 & GTGGAAAGGACGAAACACCGtattggagggcgtggtattaGTTTTAGAGCTAGAAATAG \\
\hline LEUTX_p_g4 & GTGGAAAGGACGAAACACCGgatattgaatggattattggGTTTTAGAGCTAGAAATAG \\
\hline LEUTX_p_g5 & GTGGAAAGGACGAAACACCGctgatgctgtgtagggcactGTTTTAGAGCTAGAAATAG \\
\hline LEUTXenh1_g1 & GTGGAAAGGACGAAACACCGctgtcagtgagcttccgggtGTTTTAGAGCTAGAAATAG \\
\hline LEUTXenh1_g2 & GTGGAAAGGACGAAACACCGttagctccacccegacctccGTTTTAGAGCTAGAAATAG \\
\hline LEUTXenh1_g3 & GTGGAAAGGACGAAACACCGccaaccetctaatcacgtctGTTTTAGAGCTAGAAATAG \\
\hline LEUTXenh1_g4 & GTGGAAAGGACGAAACACCGtcaggatgagggctgctcacGTTTTAGAGCTAGAAATAG \\
\hline LEUTXenh1_g5 & GTGGAAAGGACGAAACACCGcatgtgaatgagatgactggGTTTTAGAGCTAGAAATAG \\
\hline LEUTXenh2_g1 & GTGGAAAGGACGAAACACCGtgtcattatctcagtatctcGTTTTAGAGCTAGAAATAG \\
\hline LEUTXenh2_g2 & GTGGAAAGGACGAAACACCGattaatccattaatccagtcGTTTTAGAGCTAGAAATAG \\
\hline LEUTXenh2_g3 & GTGGAAAGGACGAAACACCGgtgattaggttaggagagctGTTTTAGAGCTAGAAATAG \\
\hline LEUTXenh2_g4 & GTGGAAAGGACGAAACACCGgggagatggggcctaatcaaGTTTTAGAGCTAGAAATAG \\
\hline LEUTXenh2_g5 & GTGGAAAGGACGAAACACCGatattcaaagcataacaagaGTTTTAGAGCTAGAAATAG \\
\hline
\end{tabular}

(b) Oligos used for quantitative real-time PCR.

\begin{tabular}{|l|l|l|}
\hline \multicolumn{1}{|c|}{ Gene name } & \multicolumn{1}{|c|}{ Forward oligo 5' to 3' } & \multicolumn{1}{c|}{ Reverse oligo 5' to 3' } \\
\hline DUX4 & AGGAAGAATACCGGGCTCTG & AGTCTCTCACCGGGCCTAG \\
\hline ZSCAN4 & CCTCCCAGACTTCCCAAGAT & TGTTCCAGCCATCTTGTTCA \\
\hline TRIM48 & CATCACTGGACTGAGGGACA & TGACTGTTGGCTTCATTGTGA \\
\hline CYCLOPHILIN G & TCTTGTCAATGGCCAACAGAG & GCCCATCTAAATGAGGAGTTG \\
\hline
\end{tabular}

